\title{
SYZYGIES OF CURVES AND THE EFFECTIVE CONE OF $\overline{\mathcal{M}}_{g}$
}

\author{
GAVRIL FARKAS
}

\section{INTRODUCTION}

The aim of this paper is to describe a systematic way of constructing effective divisors on $\overline{\mathcal{M}}_{g}$ having exceptionally small slope. In particular, these divisors provide a string of counterexamples to the Harris-Morrison Slope Conjecture (cf. [HMo|). In a previous paper $|\mathrm{FP}|$, we showed that the divisor $\overline{\mathcal{K}}_{10}$ on $\overline{\mathcal{M}}_{10}$ consisting of sections of $K 3$ surfaces contradicts the Slope Conjecture on $\overline{\mathcal{M}}_{10}$. Since the moduli spaces $\overline{\mathcal{M}}_{g}$ are known to behave erratically for small $g$ and since the condition that a curve of genus $g$ lie on a $K 3$ surface is divisorial only for $g=10$, the question remained whether $\overline{\mathcal{K}}_{10}$ is an isolated example or the first in a series of counterexamples. Here we prove that any effective divisor on $\overline{\mathcal{M}}_{g}$ consisting of curves satisfying a Green-Lazarsfeld syzygy type condition for a linear system residual to a pencil of minimal degree, violates the Slope Conjecture. A consequence of the existence of these effective divisors is that various moduli spaces $\overline{\mathcal{M}}_{g, n}$ with $g \leq 22$, are proved to be of general type.

We recall that the slope $s(D)$ of an effective divisor $D$ on $\overline{\mathcal{M}}_{g}$ is defined as the smallest rational number $a / b \geq 0$ such that the divisor class $a \lambda-b\left(\delta_{0}+\cdots+\delta_{[g / 2]}\right)-[D]$ is an effective combination of boundary divisors. The Slope Conjecture predicts that $s(D) \geq 6+12 /(g+1)$ for all effective divisors $D$ on $\overline{\mathcal{M}}_{g}$ (cf. [HMo]). More generally, the question of finding a good lower bound for the slope of $\overline{\mathcal{M}}_{g}$

$$
s_{g}:=\inf \left\{s(D): D \in \operatorname{Eff}\left(\overline{\mathcal{M}}_{g}\right)\right\}
$$

is of great interest for a variety of reasons, for instance it would provide a new geometric solution to the Schottky problem. In a different direction, since $s\left(K_{\overline{\mathcal{M}}_{g}}\right)=13 / 2$ (cf. [HM]), to prove that $\overline{\mathcal{M}}_{g}$ is of general type it suffices to exhibit a single effective divisor $D$ on $\overline{\mathcal{M}}_{g}$ of slope $s(D)<13 / 2$.

The Slope Conjecture is true for all $\overline{\mathcal{M}}_{g}$ with $g \leq 9$ but in [FP] we proved that on $\overline{\mathcal{M}}_{10}$, we have the equality $s\left(\overline{\mathcal{K}}_{10}\right)=7<6+12 / 11$ (in fact $\overline{\mathcal{K}}_{10}$ is the only effective divisor on $\overline{\mathcal{M}}_{10}$ having slope $\left.<6+12 / 11\right)$. In $|\mathrm{FP}|$ we also showed that $\mathcal{K}_{10}$ has four incarnations as a geometric subvariety of $\mathcal{M}_{10}$. In particular, $\mathcal{K}_{10}$ can be thought of as either (1) the locus of curves $[C] \in \mathcal{M}_{10}$ for which the rank 2 Mukai type Brill-Noether locus

$$
S U_{2}\left(C, K_{C}, 6\right):=\left\{E \in S U_{2}\left(C, K_{C}\right): h^{0}(C, E) \geq 7\right\}
$$

is $\neq \emptyset$, or (2) the locus of curves $[C] \in \mathcal{M}_{10}$ carrying a pencil $A \in W_{6}^{1}(C)$ such that the multiplication map $\mu_{A}: \operatorname{Sym}^{2} H^{0}\left(C, K_{C} \otimes A^{\vee}\right) \rightarrow H^{0}\left(C,\left(K_{C} \otimes A^{\vee}\right)^{\otimes 2}\right)$ is not surjective.

Research partially supported by the NSF Grant DMS-0450670 and by the Sloan Foundation. 
The geometric conditions (1) and (2), unlike the original definition of $\mathcal{K}_{10}$, can be extended to other genera. We fix $g=2 k-2$ and denote by $\sigma: \mathfrak{G}_{k}^{1} \rightarrow \mathcal{M}_{g}$ the Hurwitz scheme of $k$-sheeted coverings of $\mathbf{P}^{1}$ of genus $g$ parametrizing pairs $(C, A)$ with $A \in W_{k}^{1}(C)$. For each $i \geq 0$ we introduce the cycle $\mathcal{U}_{g, i}$ consisting of pairs $(C, A) \in \mathfrak{G}_{k}^{1}$ such that $K_{C} \otimes A^{\vee}$ fails the Green-Lazarsfeld property $\left(N_{i}\right)$. By setting $\mathcal{Z}_{g, i}:=\sigma\left(\mathcal{U}_{g, i}\right)$ we obtain an induced geometric stratification of $\mathcal{M}_{g}$

$$
\mathcal{Z}_{g, 0} \subset \mathcal{Z}_{g, 1} \subset \ldots \subset \mathcal{Z}_{g, i} \subset \ldots \subset \mathcal{M}_{g}
$$

If for each $(C, A) \in \mathfrak{G}_{k}^{1}$ we consider the map $C \stackrel{\left|K_{C} \otimes A^{\vee}\right|}{\longrightarrow} \mathbf{P}^{k-2}$ induced by the residual linear system, we can define two vector bundles $\mathcal{A}$ and $\mathcal{B}$ on $\mathfrak{G}_{k}^{1}$ such that

$$
\mathcal{A}(C, A)=H^{0}\left(\Omega_{\mathbf{P}^{k-2}}^{i}(i+2)\right) \text { and } \mathcal{B}(C, A)=H^{0}\left(\Omega_{\mathbf{P}^{k-2}}^{i}(i+2) \otimes \mathcal{O}_{C}\right) .
$$

For $g=6 i+10$ (hence $k=3 i+6$ ), it turns out that $\operatorname{rank}(\mathcal{A})=\operatorname{rank}(\mathcal{B})$ and $\mathcal{U}_{g, i}$ is the degeneracy locus of the natural vector bundle map $\phi: \mathcal{A} \rightarrow \mathcal{B}$, that is, $\mathcal{Z}_{g, i}$ is a virtual divisor on $\mathcal{M}_{g}$, with virtual class $\sigma_{*} c_{1}(\mathcal{B}-\mathcal{A})$. The main result of this paper is the computation of the compactification inside $\overline{\mathcal{M}}_{g}$ of this degeneracy locus (see Theorem 4.1 for a more precise statement):

Theorem A. If $\sigma: \overline{\mathfrak{G}}_{3 i+6}^{1} \rightarrow \overline{\mathcal{M}}_{6 i+10}$ is the compactification of the Hurwitz scheme by limit linear series, then there is a natural extension of the vector bundle map $\phi: \mathcal{A} \rightarrow \mathcal{B}$ over $\overline{\mathfrak{G}}_{3 i+6}^{1}$ such that $\overline{\mathcal{Z}}_{g, i}$ is the degeneracy locus of $\phi$. Moreover the class of the pushforward to $\overline{\mathcal{M}}_{g}$ of the virtual degeneracy locus of $\phi$ is given by

$$
\sigma_{*}\left(c_{1}(\mathcal{B})-c_{1}(\mathcal{A})\right) \equiv a \lambda-b_{0} \delta_{0}-b_{1} \delta_{1}-\cdots-b_{3 i+5} \delta_{3 i+5},
$$

where $a, b_{0}, \ldots, b_{3 i+5}$ are explicitly determined coefficients such that $b_{j} \geq b_{0}$ for $j \geq 1$ and

$$
\frac{a}{b_{0}}=\frac{3(4 i+7)\left(6 i^{2}+19 i+12\right)}{(i+2)\left(12 i^{2}+31 i+18\right)}<6+\frac{12}{g+1} \text {. }
$$

Corollary. If the degeneracy locus $\mathcal{Z}_{6 i+10, i}$ is an actual divisor on $\mathcal{M}_{6 i+10}$, then we have that

$$
s\left(\overline{\mathcal{Z}}_{6 i+10, i}\right)=\frac{3(4 i+7)\left(6 i^{2}+19 i+12\right)}{(i+2)\left(12 i^{2}+31 i+18\right)}<6+\frac{12}{g+1},
$$

thus contradicting the Slope Conjecture on $\overline{\mathcal{M}}_{6 i+10}$.

The idea of the proof is to define a whole host of vector bundles $\mathcal{G}_{a, b}$ and $\mathcal{H}_{a, b}$ over $\mathfrak{G}_{k}^{1}$ for $a, b \geq 0$, having fibres

$$
\mathcal{G}_{a, b}(C, A)=H^{0}\left(\Omega_{\mathbf{P}^{k-2}}^{a}(a+b) \otimes \mathcal{O}_{C}\right) \text { and } \mathcal{H}_{a, b}(C, A)=H^{0}\left(\Omega_{\mathbf{P}^{k-2}}^{a}(a+b)\right) .
$$

These bundles are related to one another by certain exact sequences (4) and (6) over $\mathfrak{G}_{k}^{1}$ (see Section 3). After an analysis over each boundary divisor $\sigma^{-1}\left(\Delta_{j}\right)$, where $0 \leq j \leq$ $3 i+5$, we find in Section 3 a unique way of extending $\mathcal{G}_{a, b}$ and $\mathcal{H}_{a, b}$ to vector bundles over $\overline{\mathfrak{G}}_{k}^{1}$ such that (4) and (6) continue to make sense and be exact and then use this to compute the Chern numbers of $\mathcal{A}=\mathcal{H}_{i, 2}$ and $\mathcal{B}=\mathcal{G}_{i, 2}$.

We expect $\mathcal{Z}_{6 i+10, i}$ to be always a divisor on $\mathcal{M}_{6 i+10}$ but we are able to check this only for small $i$. To verify this in general one would have to prove that if $[C] \in \mathcal{M}_{6 i+10}$ 
is a general curve, then one (or equivalently all) of the finitely many linear systems $\mathfrak{g}_{9 i+12}^{3 i+4}=K_{C}\left(-\mathfrak{g}_{3 i+6}^{1}\right)$ satisfies property $\left(N_{i}\right)$. This is a direct generalization of Green's Conjecture to the case of curves and line bundles $L$ with $h^{1}(L)=2$ (see Section 2 for more on this analogy).

For $g=10$ we recover of course the results from $|\mathrm{FP}|$. In the next two cases, $g=16$ and 22 we have complete results:

Theorem 1.1. The following subvariety of $\mathcal{M}_{16}$

$\mathcal{Z}_{16,1}:=\left\{[C] \in \mathcal{M}_{16}: \exists L \in W_{21}^{7}(C)\right.$ such that $C \stackrel{|L|}{\hookrightarrow} \boldsymbol{P}^{7}$ is not cut out by quadrics $\}$, is an effective divisor, and the class of its compactification is given by the formula

$$
\overline{\mathcal{Z}}_{16,1} \equiv 286\left(407 \lambda-61 \delta_{0}-325 \delta_{1}-b_{2} \delta_{2}-\cdots-b_{8} \delta_{8}\right),
$$

where $b_{j} \geq b_{1}$ for all $2 \leq j \leq 8$. In particular $s\left(\overline{\mathcal{Z}}_{16,1}\right)=407 / 61=6.6721 \ldots<6+12 / 17=$ $6.705 \ldots$, hence $\overline{\mathcal{Z}}_{16,1}$ provides a counterexample to the Slope Conjecture on $\overline{\mathcal{M}}_{16}$.

In a similar manner we have the following example of a geometric divisor on $\overline{\mathcal{M}}_{22}$ of very small slope:

Theorem 1.2. The following subvariety of $\mathcal{M}_{22}$

$$
\mathcal{Z}_{22,2}:=\left\{[C] \in \mathcal{M}_{22}: \exists L \in W_{30}^{10}(C) \text { such that } C \stackrel{|L|}{\hookrightarrow} \boldsymbol{P}^{10} \text { fails property }\left(N_{2}\right)\right\},
$$

is an effective divisor on $\mathcal{M}_{22}$ and the class of its compactification is given by the formula

$$
\overline{\mathcal{Z}}_{22,2} \equiv 25194\left(1665 \lambda-256 \delta_{0}-1407 \delta_{1}-b_{2} \delta_{2}-\cdots-b_{11} \delta_{11}\right) \text {, }
$$

where $b_{j} \geq b_{1}$ for all $2 \leq j \leq 11$. In particular $s\left(\overline{\mathcal{Z}}_{22,2}\right)=1665 / 256=6.50390 \ldots<$ $6+12 / 23=6.52173 \ldots$, and $\overline{\mathcal{Z}}_{22,2}$ gives a counterexample to the Slope Conjecture on $\overline{\mathcal{M}}_{22}$.

We note that while Theorem A gives a sequence of (virtual) examples of divisors contradicting the Slope Conjecture, for any given $g$ one can construct other (actual) divisors on $\overline{\mathcal{M}}_{g}$ of slope $<6+12 /(g+1)$ also defined in terms of syzygies. For instance, the locus consisting of curves $[C] \in \overline{\mathcal{M}}_{20}$ for which there exists $L \in W_{20}^{4}(C)$ such that $C \stackrel{|L|}{\hookrightarrow} \mathbf{P}^{4}$ is not cut out by quartics (or equivalently, the map $I_{4}(L) \otimes H^{0}(L) \rightarrow I_{5}(L)$ is not bijective), is a divisor on $\overline{\mathcal{M}}_{20}$ violating the Slope Conjecture.

We record the following consequence of Theorems 1.1 and 1.2

Theorem 1.3. (1) The moduli space of n-pointed curves $\overline{\mathcal{M}}_{22, n}$ is of general type for all $n \geq 2$.

(2) The moduli space $\overline{\mathcal{M}}_{21, n}$ is of general type for all $n \geq 4$.

(3) The moduli space $\overline{\mathcal{M}}_{16, n}$ is of general type for all $n \geq 9$.

(4) The moduli space $\overline{\mathcal{M}}_{20, n}$ is of general type for $n \geq 6$.

It is also possible to examples of divisors of small slope on the moduli spaces $\overline{\mathcal{M}}_{g, n}$ of $n$-pointed stable curves. We have only pursued this for genera $g \leq 22$ with the goal of proving that various moduli spaces $\overline{\mathcal{M}}_{g, n}$ are of general type (this is automatic for $g \geq 23$ ). One of our examples is the following: 
Theorem 1.4. The following subvariety of $\overline{\mathcal{M}}_{14,1}$

$$
\mathcal{Z}_{14,0}^{1}:=\left\{[C, p] \in \mathcal{M}_{14,1}: \exists L \in W_{18}^{6}(C) \text { such that } C \stackrel{|L(-p)|}{\longrightarrow} P^{5} \text { fails property }\left(N_{0}\right)\right\}
$$

is an effective divisor on $\mathcal{M}_{14,1}$. The class of its compactification $\overline{\mathcal{Z}}_{14,0}^{1}$ is given by the formula

$$
\overline{\mathcal{Z}}_{14,0}^{1} \equiv 33\left(237 \lambda+14 \psi-35 \delta_{0}-169 \delta_{1}-183 \delta_{13}-b_{2} \delta_{2}-\cdots-b_{12} \delta_{12}\right),
$$

where $b_{j} \geq 15+27 j$ for $3 \leq j \leq 12, j \neq 4, b_{2} \geq 325$ and $b_{4} \geq 271$. In particular $\left[\overline{\mathcal{Z}}_{14,0}^{1}\right]$ lies outside the cone of $\operatorname{Pic}\left(\overline{\mathcal{M}}_{14,1}\right)$ spanned by pullbacks of effective divisors from $\overline{\mathcal{M}}_{14}$, the boundary divisors $\delta_{0}, \ldots, \delta_{13}$ and the Weierstrass divisor $\overline{\mathcal{W}}$ on $\overline{\mathcal{M}}_{14,1}$.

An equivalent formulation of the Slope Conjecture on $\overline{\mathcal{M}}_{g}$ for $g$ such that $g+1$ is composite (which I learned from S. Keel), is to say that the Brill-Noether divisors $\overline{\mathcal{M}}_{g, d}^{r}$ consisting of curves with a $\mathfrak{g}_{d}^{r}$ when $g-(r+1)(g-d+r)=-1$, lie on a face of the effective cone $\operatorname{Eff}\left(\overline{\mathcal{M}}_{g}\right)$. It is well-known that $s\left(\overline{\mathcal{M}}_{g, d}^{r}\right)=6+12 /(g+1)$ (cf. [EH3]), so the Slope Conjecture singles out these divisors as being of minimal slope. One can ask a similar question on $\overline{\mathcal{M}}_{g, 1}$. Are the pullbacks $\pi^{*}\left(\overline{\mathcal{M}}_{g, d}^{r}\right)$ of the Brill-Noether divisors from $\overline{\mathcal{M}}_{g}$ and the Weierstrass divisor $\overline{\mathcal{W}}:=\left\{[C, p] \in \overline{\mathcal{M}}_{g, 1}: p \in C\right.$ is a Weierstrass point $\}$ on a face of the effective cone $\operatorname{Eff}\left(\overline{\mathcal{M}}_{g, 1}\right)$ ? The question makes sense especially since in [EH2] it is proved that the class of any generalized Brill-Noether divisor on $\overline{\mathcal{M}}_{g, 1}$ (that is, any codimension 1 locus of curves $(C, p) \in \overline{\mathcal{M}}_{g, 1}$ having a linear series with special ramification at $p)$ lies inside the cone of $\operatorname{Pic}\left(\overline{\mathcal{M}}_{g, 1}\right)$ spanned by by $\left[\pi^{*}\left(\overline{\mathcal{M}}_{g, d}^{r}\right)\right]$ and $[\overline{\mathcal{W}}]$. Theorem 1.4 shows that at least for $g \geq 14$ the answer to the question raised above is emphatically negative.

Acknowledgments: I had useful conversations with many people on subjects related to this paper. I especially benefitted from discussions with J. Harris, S. Keel, D. Khosla and M. Popa.

\section{SYZYGIES OF ALGEBRAIC CURVES}

In this paragraph we review a few facts about the resolution of the ideal of a curve embedded in a projective space. As a general reference for syzygies and Koszul cohomology we recommend $[\mathrm{L}]$ and $[\mathrm{GL}]$.

Suppose that $C$ is a smooth curve of genus $g$ and $L$ is a very ample line bundle on $C$ giving an embedding $C \rightarrow \mathbf{P}^{r}=\mathbf{P}(V)$, where $V=H^{0}(L)$. We denote by $I_{C / \mathbf{P}^{r}}$ the ideal of $C$ in $\mathbf{P}^{r}$ and consider its minimal resolution of free $S=\operatorname{Sym}(V)$-modules

$$
0 \rightarrow F_{r+1} \rightarrow \cdots \rightarrow F_{2} \rightarrow F_{1} \rightarrow I_{C / \mathbf{P}^{r}} \rightarrow 0 .
$$

Then one can write $F_{j}=\oplus_{l \in \mathbb{Z}} S(-j-l)^{b_{j, l}(C)}$, where $b_{j, l}(C)=\operatorname{dim}_{\mathbb{C}} \operatorname{Tor}_{j}^{S}(S(C), \mathbb{C})_{j+l}$ is the graded Betti number of $C$ that comes on the $l$-th row and $j$-th column in the Betti diagram of $C$. Following Green and Lazarsfeld we say that the pair $(C, L)$ satisfy the property $\left(N_{i}\right)$ for some integer $i \geq 1$, if $F_{j}=\oplus S(-j-1)$ for all $j \leq i$ (or equivalently in terms of graded Betti numbers, $b_{i, l}(C)=0$ for all $l \geq 2$ ). Using the computation of 
$b_{j, l}(C)$ in terms of Koszul cohomology, there is a well-known cohomological interpretation of property $\left(N_{i}\right)$ : we denote by $M=\Omega_{\mathbf{P}^{r}}(1)$ and $M_{L}=M \otimes \mathcal{O}_{C}$, hence we have an exact sequence

$$
0 \rightarrow M_{L} \rightarrow H^{0}(L) \otimes \mathcal{O}_{C} \rightarrow L \rightarrow 0 .
$$

By taking exterior powers, for each $i \geq 0$ we obtain the exact sequence:

$$
0 \rightarrow \wedge^{i+1} M_{L} \rightarrow \wedge^{i+i} H^{0}(L) \otimes \mathcal{O}_{C} \rightarrow \wedge^{i} M_{L} \otimes L \rightarrow 0 .
$$

If $L$ is a normally generated line bundle on $C$, then $(C, L)$ satisfies property $\left(N_{i}\right)$ if and only if the natural map

$$
u_{i}: \wedge^{i+1} H^{0}(L) \otimes H^{0}(L) \rightarrow H^{0}\left(\wedge^{i} M_{L} \otimes L^{2}\right)
$$

obtained by tensoring the sequence (1) and taking global sections, is surjective (cf. e.g. [GL], Lemma 1.10).

We will be interested in the vector bundle $M_{L}$ in the case when $C$ is a curve of genus $g=2 k-2$ and $L=K_{C} \otimes A^{\vee}$ is the linear series residual to a base point free pencil $A \in W_{k}^{1}(C)$. Thus $h^{0}(L)=k-1, h^{1}(L)=2$ and $\operatorname{deg}(L)=3 k-6$, hence $\mu\left(M_{L}\right)=-3$. We start by investigating the stability of the vector bundle $M_{L}$ :

Lemma 2.1. Let $C$ be a smooth curve of genus $g=2 k-2$ or $g=2 k-3$ with $k \geq 6$ and which does not possess any linear series of type $\mathfrak{g}_{3 i-1}^{i}$ for some $1 \leq i \leq k-2$ (in particular, $[C]$ lies outside a codimension $\geq 2$ locus in $\mathcal{M}_{g}$ ). Then the vector bundle $M_{L}$ is stable for each globally generated line bundle $L \in W_{3 k-6}^{k-2}(C)$.

Proof. Since we are in characteristic zero, it is enough to show that $M_{L}$ is cohomologically stable, that is, for any $i \leq k-3$ and any line bundle $E$ on $C$ of degree $e$ such that $e>\mu\left(\wedge^{i} M_{L}\right)=-3 i$ we have that $H^{0}\left(\wedge^{i} M_{L} \otimes E^{\vee}\right)=0$. We fix such a line bundle $E$ on $C$. Following a filtration argument originally due to Lazarsfeld (cf. [L]), we can choose general points $x_{1}, \ldots, x_{k-3} \in C$ such that $h^{0}\left(C, L \otimes \mathcal{O}_{C}\left(-x_{1}-\cdots-x_{k-3}\right)\right)=2$ and for which we have an exact sequence

$$
0 \longrightarrow L^{\vee}\left(x_{1}+\cdots+x_{k-3}\right) \longrightarrow M_{L} \longrightarrow \oplus_{j=1}^{k-3} \mathcal{O}_{C}\left(-x_{j}\right) \longrightarrow 0 .
$$

Taking exterior powers, we can write the exact sequence

$0 \rightarrow \wedge^{i-1}\left(\oplus_{j=1}^{k-3} \mathcal{O}_{C}\left(-x_{j}\right)\right) \otimes L^{\vee}\left(x_{1}+\cdots+x_{k-3}\right) \longrightarrow \wedge^{i} M_{L} \longrightarrow \wedge^{i}\left(\oplus_{j=1}^{k-3} \mathcal{O}_{C}\left(-x_{j}\right)\right) \longrightarrow 0$.

After we tensor this sequence by $E^{\vee}$, in order to conclude that $H^{0}\left(\wedge^{i} M_{L} \otimes E^{\vee}\right)=0$, it suffices to show that (1) $H^{0}\left(E^{\vee}\left(-D_{i}\right)\right)=0$ for each effective divisor $D_{i} \in C_{i}$ with support in $\left\{x_{1}, \ldots, x_{k-3}\right\}$, and that $(2) H^{0}\left(E^{\vee} \otimes L^{\vee}\left(-D_{k-2-i}\right)\right)=0$, for any effective divisor $D_{k-2-i} \in C_{k-2-i}$ with support in $\left\{x_{1}, \ldots, x_{k-3}\right\}$. The vanishing (2) follows because of degree reasons while the only case when (1) fails is when $h^{0}\left(E^{\vee}\right) \geq i+1$. Then $C$ carries a $\mathfrak{g}_{3 i-1}^{i}$ which we assumed not to be the case.

For later use we record the following result which can be proved in a way identical to how we proved Lemma 2.1.

Proposition 2.2. Let $C$ be a curve of genus $g$ and $L$ a globally generated $\mathfrak{g}_{d}^{r}$ on $C$. If $p \geq 1$ is an integer such that $g+\min \{r, p\} \leq d$, then for all $j \geq 2$ and $0 \leq i \leq p+2-j$, we have that $H^{1}\left(\wedge^{i} M_{L} \otimes L^{\otimes j}\right)=0$. 
Remark 2.3. When $p \geq r$, the condition $g+\min \{p, r\} \leq d$ is equivalent with $H^{1}(L)=0$.

We fix $g=2 k-2$ where $k \geq 3$. We denote by $\mathcal{M}_{g}^{0}$ the open subvariety of $\mathcal{M}_{g}$ consisting of curves $C$ of genus $g$ which carry no $\mathfrak{g}_{k-1}^{1}$ 's and which have no automorphisms (clearly the complement of $\mathcal{M}_{g}^{0}$ inside $\mathcal{M}_{g}$ has codimension $\geq 2$ ). Over $\mathcal{M}_{g}^{0}$ we have a universal curve $\pi: \mathcal{C} \rightarrow \mathcal{M}_{g}^{0}$ and we also consider the Hurwitz scheme $\sigma: \mathfrak{G}_{k}^{1} \rightarrow \mathcal{M}_{g}^{0}$ parametrising pairs $(C, A)$, where $[C] \in \mathcal{M}_{g}^{0}$ and $A \in W_{k}^{1}(C)$ is a (necessarily base point free) $\mathfrak{g}_{k}^{1}$ on $C$. It is a classical result that $\mathfrak{G}_{k}^{1}$ is a smooth irreducible variety which is finite over $\mathcal{M}_{g}^{0}$. The isomorphism $\mathfrak{G}_{k}^{1} \ni(C, A) \mapsto\left(C, K_{C} \otimes A^{\vee}\right) \in \mathfrak{G}_{3 k-6}^{k-2}$ will be used throughout the paper.

Let us consider now an element $(C, A) \in \mathfrak{G}_{k}^{1}$ and set $L:=K_{C} \otimes A^{\vee}$. The genericity assumption on $C$ implies that $L$ is very ample and gives an embedding $C \subset \mathbf{P}^{k-2}$ of degree $3 k-6$. We denote by $\mathcal{I}_{C}$ the ideal sheaf of $C$ in this embedding.

Proposition 2.4. Fix integers $g=2 k-2$ with $k \geq 3$ and $i \geq 0$ with $2 k \geq 3 i+7$. If $(C, A) \in \mathfrak{G}_{k}^{1}$ and $L=K_{C} \otimes A^{\vee}$, then $(C, L)$ fails to satisfy property $\left(N_{i}\right)$ if and only if

$$
h^{0}\left(\boldsymbol{P}^{k-2}, \wedge^{i} M_{\boldsymbol{P}^{k-2}} \otimes \mathcal{I}_{C}(2)\right) \geq\left(\begin{array}{c}
k-2 \\
i+1
\end{array}\right)(k-3 i-6)+2\left(\begin{array}{c}
k-1 \\
i+1
\end{array}\right)-\left(\begin{array}{c}
k-1 \\
i+2
\end{array}\right)+1 .
$$

Proof. We use the following diagram of exact sequences for the embedding $C \stackrel{|L|}{\hookrightarrow} \mathbf{P}^{k-2}$ :

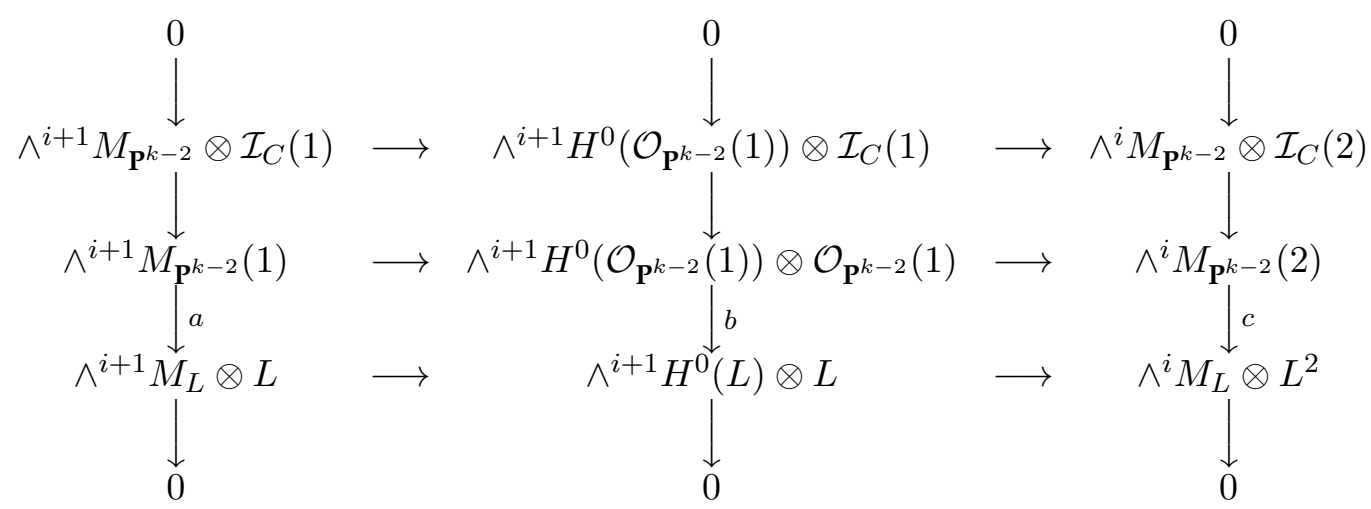

Since $H^{1}\left(\mathbf{P}^{k-2}, \wedge^{i+1} M_{\mathbf{P}^{k-2}}(1)\right)=0$ (e.g. because of Bott vanishing), we can apply the snake lemma to the diagram obtained by taking global sections in the two lower rows. The map induced by $b$ is an isomorphism, which gives an isomorphism

$$
H^{0}\left(\mathbf{P}^{k-2}, \wedge^{i} M_{\mathbf{P}^{k-2}} \otimes \mathcal{I}_{C}(2)\right) \cong H^{1}\left(\mathbf{P}^{k-2}, \wedge^{i+1} M_{\mathbf{P}^{k-2}} \otimes \mathcal{I}_{C}(1)\right) .
$$

Since $M_{L}$ is a stable bundle, we have that $H^{1}\left(\wedge^{i} M_{L} \otimes L^{2}\right)=0$ based on slope considerations, and thus $C$ satisfies $\left(N_{i}\right)$ if and only if the map

$$
H^{1}\left(\wedge^{i+1} M_{L} \otimes L\right) \rightarrow \wedge^{i+1} H^{0}(L) \otimes H^{1}(L)
$$


is an isomorphism, or equivalently $h^{1}\left(\wedge^{i+1} M_{L} \otimes L\right) \leq h^{1}\left(\wedge^{i+1} H^{0}(L) \otimes L\right)=2\left(\begin{array}{c}k-1 \\ i+1\end{array}\right)$.

From Riemann-Roch, this is the same thing as having

$$
\operatorname{dim}(\operatorname{Coker}(a)) \leq\left(\begin{array}{c}
k-2 \\
i+1
\end{array}\right)(k-3 i-6)+2\left(\begin{array}{c}
k-1 \\
i+1
\end{array}\right)-\left(\begin{array}{c}
k-1 \\
i+2
\end{array}\right) .
$$

But $\operatorname{Coker}(a) \cong H^{0}\left(\wedge^{i} M_{\mathbf{P}^{k-2}} \otimes \mathcal{I}_{C}(2)\right)$, so failure of $\left(N_{i}\right)$ is equivalent to the map $c$ having a kernel of dimension $\geq$

$$
\left(\begin{array}{c}
k-2 \\
i+1
\end{array}\right)(k-3 i-6)+2\left(\begin{array}{c}
k-1 \\
i+1
\end{array}\right)-\left(\begin{array}{c}
k-1 \\
i+2
\end{array}\right)+1=\left(\begin{array}{c}
k-2 \\
i+1
\end{array}\right)(k-3 i-6)\left(1-\frac{1}{(k-i-2)(i+2)}\right)+1 .
$$

Remark 2.5. An immediate consequence of this proof is that when $k-3 i-6<0$ (or equivalently $g \leq 6 i+8)$, the pair $(C, A)$ always fails property $\left(N_{i}\right)$. When $k=3 i+6$ we have that $(C, A)$ fails $\left(N_{i}\right)$ if and only if $h^{0}\left(\mathbf{P}^{k-2}, \wedge^{i} M_{\mathbf{P}^{k-2}} \otimes \mathcal{I}_{C}(2)\right) \geq 1$, which we expect to be a divisorial condition on $\mathfrak{G}_{k}^{1}$.

This proposition allows us to define a determinantal subscheme of $\mathfrak{G}_{k}^{1}$ consisting of pairs $(C, A)$ for which $L$ fails to satisfy property $\left(N_{i}\right)$. On the fibre product $\mathcal{C} \times \mathcal{M}_{g}^{0} \mathfrak{G}_{k}^{1}$ there is a universal Poincare bundle $\mathcal{L}$ whose existence is guaranteed by the universal property of $\mathfrak{G}_{k}^{1}$. If $p_{1}: \mathcal{C} \times{ }_{\mathcal{M}_{g}^{0}} \mathfrak{G}_{k}^{1} \rightarrow \mathcal{C}$ and $p_{2}: \mathcal{C} \times{ }_{\mathcal{M}_{g}^{0}} \mathfrak{G}_{k}^{1} \rightarrow \mathfrak{G}_{k}^{1}$ are the natural projections, then $\mathcal{E}:=p_{2 *}\left(p_{1}^{*} \omega_{\pi} \otimes \mathcal{L}^{\vee}\right)$ is a vector bundle of rank $k-1$ with fibre $\mathcal{E}(C, A)=$ $H^{0}\left(C, K_{C} \otimes A^{\vee}\right)$ over each point $(C, A) \in \mathfrak{G}_{k}^{1}$. We have a tautological embedding of the pullback of the universal curve $\mathcal{C} \times{ }_{\mathcal{M}_{g}^{0}} \mathfrak{G}_{k}^{1}$ into the projective bundle $u: \mathbf{P}(\mathcal{E}) \rightarrow \mathfrak{G}_{k}^{1}$ and we denote by $\mathcal{J} \subset \mathcal{O}_{\mathbf{P}(\mathcal{E})}$ the ideal sheaf of the image. Next, we define the vector bundle $\mathcal{N}$ on $\mathbf{P}(\mathcal{E})$ by the sequence

$$
0 \longrightarrow \mathcal{N} \rightarrow u^{*}(\mathcal{E}) \longrightarrow \mathcal{O}_{\mathbf{P}(\mathcal{E})}(1) \longrightarrow 0
$$

and we further introduce two vector bundles $\mathcal{A}$ and $\mathcal{B}$ over $\mathfrak{G}_{k}^{1}$ by setting

$$
\mathcal{A}:=u_{*}\left(\wedge^{i} \mathcal{N} \otimes \mathcal{O}_{\mathbf{P}(E)}(2)\right), \text { and } \mathcal{B}:=u_{*}\left(\wedge^{i} \mathcal{N} \otimes \mathcal{O}_{\mathcal{C} \times{ }_{\mathcal{M}_{g}^{0}} \mathfrak{G}_{k}^{1}}(2)\right)
$$

If $C \subset \mathbf{P}^{k-2}$ is the embedding given by $L=K_{C} \otimes A^{\vee}$, then

$$
\mathcal{A}(C, A)=H^{0}\left(\mathbf{P}^{k-2}, \wedge^{i} M_{\mathbf{P}^{k-2}}(2)\right) \text { and } \mathcal{B}(C, A)=H^{0}\left(C, \wedge^{i} M_{L} \otimes L^{2}\right)
$$

and there is a natural vector bundle morphism $\phi: \mathcal{A} \rightarrow \mathcal{B}$. From Grauert's Theorem we see that both $\mathcal{A}$ and $\mathcal{B}$ are vector bundles over $\mathfrak{G}_{k}^{1}\left(\right.$ for $\mathcal{B}$ use that $H^{1}\left(C, \wedge^{i} M_{L} \otimes L^{2}\right)=0$ because $M_{L}$ is stable). Moreover from Bott's Theorem and Riemann-Roch respectively, we obtain that

$$
\operatorname{rank}(\mathcal{A})=(i+1)\left(\begin{array}{c}
k \\
i+2
\end{array}\right) \text { and } \operatorname{rank}(\mathcal{B})=\left(\begin{array}{c}
k-2 \\
i
\end{array}\right)(4 k-9-3 i) .
$$

Then Proposition 2.4 can be restated as follows:

Proposition 2.6. The cycle

$$
\mathcal{U}_{g, i}:=\left\{(C, A) \in \mathfrak{G}_{k}^{1}:\left(C, K_{C} \otimes A^{\vee}\right) \text { fails property }\left(N_{i}\right)\right\},
$$

can be realized as the degeneracy locus of rank $(i-1)\left(\begin{array}{c}k-1 \\ i+1\end{array}\right)+(i+2)\left(\begin{array}{c}k-1 \\ i+2\end{array}\right)-\left(\begin{array}{c}k-2 \\ i+1\end{array}\right)(k-3 i-6)-1$ of the vector bundle map $\phi: \mathcal{A} \rightarrow \mathcal{B}$ over $\mathfrak{G}_{k}^{1}$. 
In this way we obtain a stratification of $\mathcal{M}_{g}^{0}$ with strata $\mathcal{Z}_{g, i}:=\sigma\left(\mathcal{U}_{g, i}\right)$ consisting of those curves $C$ for which there exists a pencil $A \in W_{k}^{1}(C)$ such that $\left(C, K_{C} \otimes A^{\vee}\right)$ fails property $\left(N_{i}\right)$. Note that when $g=6 i+10$ then $\operatorname{rank}(\mathcal{A})=\operatorname{rank}(\mathcal{B})$ and $\mathcal{U}_{g, i}$ is simply the degeneracy locus of $\phi$ and we expect $\mathcal{Z}_{g, i}$ to be a divisor on $\mathcal{M}_{g}^{0}$.

It is of course very natural to compare this newly defined stratification

$$
\mathcal{Z}_{g, 0} \subset \mathcal{Z}_{g, 1} \subset \ldots \subset \mathcal{Z}_{g, i} \subset \ldots \subset \mathcal{M}_{g}^{0}
$$

with the more classical stratification of $\mathcal{M}_{g}$ defined in terms of syzygies of $K_{C}$. If for each $i \geq 0$ we set

$$
\mathcal{N}_{g, i}:=\left\{[C] \in \mathcal{M}_{g}:\left(C, K_{C}\right) \text { fails property }\left(N_{i}\right)\right\},
$$

then Green's Conjecture for generic curves of fixed gonality (cf. |V3], |V2]) can be read as saying that for all $0 \leq i \leq(g-2) / 2$, the locus $\mathcal{N}_{g, i}$ coincides with the $(i+2)$-gonal locus $\mathcal{M}_{g, i+2}^{1}$ of curves with a $\mathfrak{g}_{i+2}^{1}$, while $\mathcal{N}_{g, i}=\mathcal{M}_{g}$ for all $i \geq(g-1) / 2$. One of the morals of this paper is that the stratification $\mathcal{Z}_{g, i}$ is very different in nature from the one given by gonality: whereas $\mathcal{N}_{g, 0}$ is the locus of hyperelliptic curves of genus $g$, the smallest stratum $\mathcal{Z}_{g, 0}$ is intimately related to the locus of curves lying on $K 3$ surfaces which is well-known to be transversal to any Brill-Noether locus (a curve of genus $g$ lying on a general $K 3$ surface satisfies the Brill-Noether Theorem, cf. [L]).

We have already seen that $\mathcal{Z}_{g, i}=\mathcal{M}_{g}^{0}$ when $g \leq 6 i+8$ (cf. Remark 2.5). A result due to Mukai and Voisin (cf. |V1], Proposition 3.2) states that if $C$ is a smooth curve of genus $g=2 k-2$ sitting on a $K 3$ surface $S$ with $\operatorname{Pic}(S)=\mathbb{Z}[C]$, then for all $A \in W_{k}^{1}(C)$ the multiplication map $\operatorname{Sym}^{2} H^{0}\left(K_{C} \otimes A^{\vee}\right) \rightarrow H^{0}\left(\left(K_{C} \otimes A^{\vee}\right)^{\otimes 2}\right)$ is not surjective, in other words, $[C] \in \mathcal{Z}_{g, 0}$. In a forthcoming paper we will show that the converse also holds, that is, the closure in $\mathcal{M}_{g}$ of the smallest stratum $\mathcal{Z}_{g, 0}$ coincides with the locus

$$
\mathcal{K}_{g}:=\left\{[C] \in \mathcal{M}_{g}: C \text { lies on a } K 3 \text { surface }\right\} .
$$

The possibility of such an equality of cycles has already been raised in Voisin's paper (cf. [V1], Remarques 4.13). Its main appeal lies in the fact that it gives an intrinsic characterization of a curve lying on a $K 3$ surface which makes no reference to the $K 3$ surface itself! We now make the following:

Conjecture 2.7. For an even genus $g \geq 6 i+10$, the stratum $\mathcal{Z}_{g, i}$ is a proper subvariety of $\mathcal{M}_{g}^{0}$. In particular, when $g=6 i+10$, the stratum $\mathcal{Z}_{g, i}$ is a divisor on $\mathcal{M}_{g}^{0}$.

For $g \leq 8$ the conjecture is trivially true because $\mathcal{K}_{g}=\mathcal{M}_{g}$, that is, every curve of (even) genus $g \leq 8$ sits on a $K 3$ surface. The first interesting case is $g=10$ when the conjecture holds and the identification $\mathcal{K}_{10}=\mathcal{Z}_{10,0}$ is part of a more general picture (see $|\mathrm{FP}|$ for details). We have checked Conjecture 2.7 for all genera $g \leq 24$ sometimes using the program Macaulay. We will describe this in detail in the most interesting cases, $g=16$ and $g=22$, when the strata $\mathcal{Z}_{16,1}$ and $\mathcal{Z}_{22,2}$ are divisors on $\mathcal{M}_{16}$ and $\mathcal{M}_{22}$ respectively.

Theorem 2.8. Let $C$ be a general curve of genus 16. Then every linear series $A \in W_{9}^{1}(C)$ gives an embedding $C \stackrel{\left|K_{C} \otimes A^{\vee}\right|}{\longrightarrow} \boldsymbol{P}^{7}$ of degree 21 such that the ideal of $C$ is generated by quadrics (that is, it satisfies property $\left(N_{1}\right)$ ). In particular $\mathcal{Z}_{16,1}$ is a divisor on $\mathcal{M}_{16}$. 
Proof. From the irreducibility of the Hurwitz scheme of coverings of $\mathbf{P}^{1}$ it follows that the variety $\mathfrak{G}_{9}^{1}=\mathfrak{G}_{16,9}^{1}$ parametrizing pairs $(C, A)$ with $[C] \in \mathcal{M}_{16}$ and $A \in W_{9}^{1}(C)$ is irreducible. To prove that $\mathcal{Z}_{16,1}$ is a divisor it is enough to exhibit an element $(C, A) \in \mathfrak{G}_{9}^{1}$ with $K_{C} \otimes A^{\vee}$ very ample, such that the embedded curve $C \stackrel{\left|K_{C} \otimes A^{\vee}\right|}{\hookrightarrow} \mathbf{P}^{7}$ is cut out by quadrics.

We consider 13 general points in $\mathbf{P}^{2}$ denoted by $p_{1}, p_{2}, q_{1}, \ldots, q_{7}$ and $r_{1}, \ldots, r_{4}$ respectively, and define the linear system

$$
H \equiv 8 h-3\left(E_{p_{1}}+E_{p_{2}}\right)-2 \sum_{i=1}^{7} E_{q_{i}}-\sum_{j=1}^{4} E_{r_{j}}
$$

on the blow-up $S=B l_{13}\left(\mathbf{P}^{2}\right)$. Here $h$ denotes the pullback of the line class from $\mathbf{P}^{2}$. Using the program Macaulay it is easy to check that $S \stackrel{|H|}{\hookrightarrow} \mathbf{P}^{7}$ is an embedding and the graded Betti diagram of $S$ is

$$
\begin{array}{cccc}
1 & - & - & - \\
- & 7 & - & - \\
- & - & 35 & 56
\end{array}
$$

Thus $S$ is cut out by quadrics. To carry out this calculation we chose the 13 points in $\mathbf{P}^{2}$ randomly using the Hilbert-Burch theorem so that they satisfy the Minimal Resolution Conjecture (see [SchT] for details on how to pick random points in $\mathbf{P}^{2}$ using Macaulay). Next we consider a curve $C \subset S$ in the linear system

$$
C \equiv 14 h-5\left(E_{p_{1}}+E_{p_{2}}\right)-4 \sum_{i=1}^{6} E_{q_{i}}-3 E_{q_{7}}-2 \sum_{j=1}^{3} E_{r_{j}}-E_{r_{4}} .
$$

By using Macaulay we pick $C$ randomly in its linear system and then check that $C$ is smooth, $g(C)=16$ and $\operatorname{deg}(C)=21$. To show that $C$ is cut out by quadrics one can either compute directly the Betti diagram of $C$ or otherwise use that since $S$ is cut out by quadrics, to conclude the same thing about $C$, it suffices to show that the map

$$
m: H^{0}(S, H) \otimes H^{0}(S, 2 H-C) \rightarrow H^{0}(S, 3 H-C)
$$

is surjective (or equivalently injective). Since $h^{0}(S, 2 H-C)=2$, from the base point free pencil trick we get that $\operatorname{Ker}(m)=H^{0}(S, C-H)=0$, because $C-H \equiv 6 h-2 E_{p_{1}}-$ $2 E_{p_{2}}-2 \sum_{i=1}^{6} E_{q_{i}}-E_{q_{7}}-\sum_{j=1}^{3} E_{r_{j}}$ is clearly not effective for a general choice of the 13 points.

Remark 2.9. A possible objection to this proof could be that while we defined the Hurwitz space $\mathfrak{G}_{9}^{1} \rightarrow \mathcal{M}_{16}^{0}$ over the open part of $\mathcal{M}_{16}$ consisting of curves carrying no $\mathfrak{g}_{8}^{1}$ 's, the curve $C$ we constructed in the proof of Theorem 2.8 might lie outside $\mathcal{M}_{16}^{0}$. However, this does not affect our conclusions because for every $g$ and $l<(g+3) / 2$, the Hurwitz space of pairs $(C, A)$ with $[C] \in \mathcal{M}_{g}$ an $A \in W_{l}^{1}(C)$, is an irreducible variety, that is, there can be no component of the Hurwitz space mapping to a proper subvariety of the l-gonal locus $\mathcal{M}_{g, l}^{1}$ (This statement can be easily proved with the methods from [AC1]). 
Remark 2.10. For later use we are going to record a slight generalization of Theorem 2.8. The conclusion of the theorem holds for the image in $\mathbf{P}^{7}$ of any plane curve $C$ that is sufficiently general in the linear system given by (2). In particular one can choose $C$ to have a cusp at a general extra point $y \in \mathbf{P}^{2}$, in which case we obtain that a general one-cuspidal curve $C \subset \mathbf{P}^{7}$ of arithmetic genus 16 and degree 21 is cut out by quadrics (this again is quite easily checked with Macaulay). Since the Hilbert scheme of these curves is irreducible (use again the Hurwitz scheme), it follows that for a general $(C, y) \in$ $\mathcal{M}_{15,1}$ and for an arbitrary linear system $L \in W_{21}^{7}(C)$ having a cusp at $y$, the ideal of the cuspidal image curve $C \stackrel{|L|}{\rightarrow} \mathbf{P}^{7}$ is generated by quadrics.

In a somewhat similar manner we are going to show that for $g=22$ the locus $\mathcal{Z}_{22,2}$ is a divisor.

Theorem 2.11. Let $C$ be a general curve of genus 22. Then for every linear series $A \in W_{12}^{1}(C)$ we have that $\left|K_{C} \otimes A^{\vee}\right|$ is very ample and the resulting embedding $A \stackrel{\left|K_{C} \otimes A^{\vee}\right|}{\hookrightarrow} P^{10}$ satisfies property $\left(N_{2}\right)$. In particular, the locus of curves $C$ of genus 22 carrying a linear series $\mathfrak{g}_{30}^{10}=$ $K_{C}\left(-\mathfrak{g}_{12}^{1}\right)$ that fails to satisfy $\left(N_{2}\right)$, is a divisor on $\mathcal{M}_{22}$.

Proof. Ideally we would like to construct a rational surface $S \subset \mathbf{P}^{10}$ which satisfies property $\left(N_{2}\right)$ and then consider a suitable curve $C \subset S$ with $g(C)=22$ and $\operatorname{deg}(C)=$ 30. For numerical reasons this will turn out not to be possible but we will be able to construct a smooth curve $C^{\prime} \subset S$ with $g\left(C^{\prime}\right)=20$ and $\operatorname{deg}\left(C^{\prime}\right)=28$ which satisfies $\left(N_{2}\right)$. The desired curve $C$ will be a smoothing in $\mathbf{P}^{10}$ of $C \cup L \cup L^{\prime}$, where $L$ and $L^{\prime}$ are general chords of $C^{\prime}$.

We start by choosing random points $p, p_{1}, \ldots, p_{7}, q_{1}, \ldots, q_{5} \in \mathbf{P}^{2}$. Then the linear system

$$
H=11 h-4 E_{p}-3 \sum_{i=1}^{7} E_{p_{i}}-2 \sum_{j=1}^{5} E_{q_{j}}
$$

yields an embedding $S \subset \mathbf{P}^{10}$ of $B l_{13}\left(\mathbf{P}^{2}\right)$. Using Macaulay we get that the upper left corner of the Betti diagram of $S$ is

$$
\begin{array}{ccccc}
1 & - & - & - & - \\
- & 29 & 98 & 72 & - \\
- & - & - & 264 & -
\end{array}
$$

that is, $S$ satisfies property $\left(N_{2}\right)$, hence $H^{1}\left(S, \wedge^{3} M_{S}(1)\right)=0$. If $\Gamma \equiv H$ is a general hyperplane section of $S$, then $g(\Gamma)=13, \operatorname{deg}(\Gamma)=22$ and an argument very similar to the one in Lemma 2.1 shows that the vector bundle $M_{\Gamma}$ is stable. In particular $H^{1}\left(\Gamma, \wedge^{2} M_{\Gamma}(2)\right)=0$. Since $M_{S \mid \Gamma} \cong M_{\Gamma} \oplus \mathcal{O}_{\Gamma}$ one can write the exact sequence

$$
0 \longrightarrow \wedge^{i} M_{S}(1) \longrightarrow \wedge^{i} M_{S}(2) \longrightarrow \wedge^{i} M_{\Gamma}(2) \oplus \wedge^{i-1} M_{\Gamma}(2) \longrightarrow 0
$$


which leads to the vanishing $H^{2}\left(S, \wedge^{3} M_{S}(1)\right)=0$. Suppose now that $C \subset S$ is a smooth non-degenerate curve. We have a commutative diagram:

$$
\begin{aligned}
H^{1}\left(C, \wedge^{3} M_{C}(1)\right) & \longrightarrow \wedge^{3} H^{0}\left(\mathcal{O}_{C}(1)\right) \otimes H^{1}\left(\mathcal{O}_{C}(1)\right) \\
H^{1}\left(\wedge^{2} M_{S}(2 H-C)\right) \hookrightarrow H^{2}\left(\wedge^{3} M_{S}^{\downarrow}(H-C)\right) & \rightarrow \wedge^{3} H^{0}\left(\mathcal{O}_{S}(1)\right) \otimes H^{2}(H-C),
\end{aligned}
$$

where the left vertical map is isomorphic because $H^{1}\left(S, \wedge^{3} M_{S}(1)\right)=H^{2}\left(S, \wedge^{3} M_{S}(1)\right)=$ 0 . To conclude that $C$ satisfies $\left(N_{2}\right)$, it suffices to show that $H^{1}\left(S, \wedge^{2} M_{S}(2 H-C)\right)=0$, or equivalently, that the map $f: \wedge^{2} H^{0}\left(\mathcal{O}_{S}(1)\right) \otimes H^{0}(2 H-C) \rightarrow H^{0}\left(M_{S}(3 H-C)\right)$ obtained from the Koszul complex, is surjective. If $(\operatorname{deg}(C), g(C))=(30,22)$, then one can check easily that $h^{0}(S, 2 H-C)=3$ and $h^{0}\left(S, M_{S}(3 H-C)\right)=207$, hence $f$ cannot be surjective for dimensional reasons. The closest we can get to these numerical invariants is when $(\operatorname{deg}(C), g(C))=(28,20)$ and this is the type of curve on $S$ we will be looking for. Take

$$
C^{\prime} \equiv 17 h-6 E_{p}-5 \sum_{i=}^{7} E_{p_{i}}-3 \sum_{j=1}^{5} E_{q_{j}},
$$

and consider the embedding $C^{\prime} \subset \mathbf{P}^{10}$ given by $|H|$. Using Macaulay we check that $C^{\prime}$ is a smooth curve of genus 20 and degree 28 with graded Betti diagram

$$
\begin{array}{cccc}
1 & - & - & - \\
- & 27 & 80 & - \\
- & - & - & 432
\end{array}
$$

Hence $C^{\prime}$ satisfies $\left(N_{2}\right)$. Now choose two general chords $L$ and $L^{\prime}$ of $C$ and define $C:=C^{\prime} \cup L \cup L^{\prime}$. Clearly $g(C)=22, \operatorname{deg}(C)=30$ and one last check with Macaulay shows that $b_{2 j}(C)=0$ for $j \geq 2$, that is, $C$ satisfies $\left(N_{2}\right)$.

Remark 2.12. Just like in the case $g=16$ we have a slight variation of the last Theorem. The same proof shows that for a general $(C, y) \in \mathcal{M}_{21,1}$ and for an arbitrary linear series $L \in W_{30}^{10}(C)$ that has a cusp at $y$, the one-cuspidal image curve $C \stackrel{|L|}{\rightarrow} \mathbf{P}^{10}$ satisfies property $\left(N_{2}\right)$.

\section{INTERSECTION THEORY CALCULATIONS ON $\overline{\mathcal{M}}_{g}$}

Recall that we have realized $\mathcal{Z}_{6 i+10, i}$ as the image of the degeneracy locus $\mathcal{U}_{6 i+10, i}$ of a vector bundle morphism $\phi: \mathcal{A} \rightarrow \mathcal{B}$ over $\mathfrak{G}_{k}^{1}$. To compute the class of the compactification $\overline{\mathcal{Z}}_{6 i+10, i}$ we are going to extend the determinantal structure of $\mathcal{Z}_{6 i+10, i}$ over the boundary divisors in $\overline{\mathcal{M}}_{6 i+10}$.

We set $g:=6 i+10, k:=3 i+6$ and denote by $\widetilde{\mathcal{M}}_{g}:=\mathcal{M}_{g}^{0} \cup\left(\cup_{j=0}^{3 i+5} \Delta_{j}^{0}\right)$ the locally closed subset of $\overline{\mathcal{M}}_{g}$ defined as the union of the locus $\mathcal{M}_{g}^{0}$ of smooth curves carrying no linear systems $\mathfrak{g}_{k-1}^{1}$ to which we add the open subsets $\Delta_{j}^{0} \subset \Delta_{j}$ for $1 \leq j \leq 3 i+5$ consisting of 1-nodal genus $g$ curves $C \cup_{y} D$, with $[C] \in \mathcal{M}_{g-j}$ and $[D, y] \in \mathcal{M}_{j, 1}$ being Brill-Noether general curves, and the locus $\Delta_{0}^{0} \subset \Delta_{0}$ containing 1-nodal irreducible genus $g$ curves $C^{\prime}=C / q \sim y$, where $[C, q] \in \mathcal{M}_{g-1}$ is a Brill-Noether general pointed 
curve and $y \in C$, together with their degenerations consisting of unions of a smooth genus $g-1$ curve and a nodal rational curve. One can then extend the finite covering $\sigma: \mathfrak{G}_{3 k-6}^{k-2} \rightarrow \mathcal{M}_{g}^{0}$ to a proper, generically finite map

$$
\sigma: \widetilde{\mathfrak{G}}_{3 k-6}^{k-2} \rightarrow \widetilde{\mathcal{M}}_{g}
$$

by letting $\widetilde{\mathfrak{G}}_{3 k-6}^{k-2}$ be the variety of limit $\mathfrak{g}_{3 k-6}^{k-2}$ 's on the treelike curves from $\widetilde{\mathcal{M}}_{g}$ (see [EH1], Theorem 3.4 for the construction of the space of limit linear series).

We will be interested in intersecting the divisors $\overline{\mathcal{Z}}_{6 i+10, i}$ on $\overline{\mathcal{M}}_{g}$ with test curves in the boundary of $\overline{\mathcal{M}}_{6 i+10}$ which are defined as follows: we fix a Brill-Noether general curve $C$ of genus $2 k-3=6 i+9$, a general point $q \in C$ and a general elliptic curve $E$. We define two 1-parameter families

(3)

$$
C^{0}:=\{C / y \sim q: y \in C\} \subset \Delta_{0} \subset \overline{\mathcal{M}}_{2 k-2} \text { and } C^{1}:=\left\{C \cup_{y} E: y \in C\right\} \subset \Delta_{1} \subset \overline{\mathcal{M}}_{2 k-2} \text {. }
$$

It is well-known that these families intersect the generators of $\operatorname{Pic}\left(\overline{\mathcal{M}}_{2 k-2}\right)$ as follows:

$$
\begin{gathered}
C^{0} \cdot \lambda=0, C^{0} \cdot \delta_{0}=-(4 k-6), C^{0} \cdot \delta_{1}=1 \text { and } C^{0} \cdot \delta_{a}=0 \text { for } a \geq 2, \text { and } \\
C^{1} \cdot \lambda=0, C^{1} \cdot \delta_{0}=0, C^{1} \cdot \delta_{1}=-(4 k-8), C^{1} \cdot \delta_{a}=0 \text { for } a \geq 2 .
\end{gathered}
$$

Next, we fix an integer $2 \leq j \leq k-1$, a general curve $C$ of genus $2 k-2-j$ and a general curve pointed curve $(D, y)$ of genus $j$. We define the 1-parameter family $C^{j}:=\left\{C \cup_{y} D: y \in C\right\} \subset \Delta_{j} \subset \overline{\mathcal{M}}_{2 k-2}$. We have that

$$
C^{j} \cdot \lambda=0, C^{j} \cdot \delta_{a}=0 \text { for } a \neq j \text { and } C^{j} \cdot \delta_{j}=-(4 k-6-2 j) .
$$

During our calculations we will often need the following enumerative result (cf. $[\mathrm{HM} \mid$, pg. 71):

Proposition 3.1. Let $C$ be a general curve of genus $j, y \in C$ a general fixed point and an integer $0 \leq \alpha \leq j / 2$.

(1) The number of pencils $A \in W_{j-\alpha+1}^{1}(Y)$ satisfying $h^{0}(Y, A(-(j+1-2 \alpha) y)) \geq 1$ is equal to

$$
a(j, \alpha)=\frac{j+1-2 \alpha}{j+1-\alpha}\left(\begin{array}{c}
j \\
\alpha
\end{array}\right) .
$$

(2) The number of pencils $A \in W_{j-\alpha}^{1}(C)$ satisfying $h^{0}(Y, A(-(j-2 \alpha) q)) \geq 1$ for some unspecified point $q \in Y$ is equal to

$$
b(j, \alpha)=(j-2 \alpha-1)(j-2 \alpha)(j-2 \alpha+1)\left(\begin{array}{l}
j \\
\alpha
\end{array}\right) .
$$

Definition 3.2. (1) For a fixed general curve $C$ of genus $2 k-3$ we denote by $\delta(k, 1)$ the (finite) number of linear series $L \in W_{3 k-6}^{k-2}(C)$ for which there exists a point $y \in C$ with $h^{0}(L(-2 y)) \geq k-2$ and $h^{0}(L(-k y)) \geq 1$.

(2) We fix $2 \leq j \leq k-1,0 \leq \alpha \leq j / 2$ and a general curve $C$ of genus $2 k-2-j$. We denote by $\delta(k, j, \alpha)$ the number of linear series $L_{C} \in W_{3 k-j-4}^{k-2}(C)$ for which there exists a point $y \in C$ such that $\left.h^{0}(L(-(\alpha+1) y)) \geq k-1-\alpha, h^{0}(L-(j-\alpha+2) y)\right) \geq k-1+\alpha-j$ and $h^{0}(L(-(k+1) y)) \geq 1$. 
The numbers $\delta(k, 1)$ and $\delta(k, j, \alpha)$ can be computed by degeneration. Since we do not need their actual values we skip this calculation. Next we describe $\widetilde{\mathfrak{G}}_{3 k-6}^{k-2}$ set theoretically. To set notation, if $X$ is a treelike curve and $l$ is a limit $\mathfrak{g}_{d}^{r}$ on $X$, for a component $Y$ of $X$ we denote by $l_{Y}=\left(L_{Y}, V_{Y} \subset H^{0}\left(L_{Y}\right)\right)$ the $Y$-aspect of $l$. For a point $y \in Y$ we denote by by $\left\{a_{s}^{l_{Y}}(C)\right\}_{s=0 \ldots r}$ the vanishing sequence of $l$ at $y$ and by $\rho\left(l_{Y}, y\right):=\rho(g, r, d)-\sum_{i=0}^{r}\left(a_{i}^{l_{Y}}(y)-i\right)$ the adjusted Brill-Noether number with respect to $y$

Proposition 3.3. (1) Let $C_{y}^{1}=C \cup_{y} E$ be an element of $\Delta_{1}^{0}$. If $\left(l_{C}, l_{E}\right)$ is a limit $\mathfrak{g}_{3 k-6}^{k-2}$ on $C_{y}^{1}$, then $V_{C}=H^{0}\left(L_{C}\right)$ and $L_{C} \in W_{3 k-6}^{k-2}(C)$ has a cusp at $y$, that is, $h^{0}(C, L(-2 y))=k-2$. If $y \in C$ is a general point, then $l_{E}=\left(\mathcal{O}_{E}((3 k-6) y),(2 k-5) y+|(k-1) y|\right)$, that is, $l_{E}$ is uniquely determined. If $y \in C$ is one of the finitely many points for which there exists $L_{C} \in W_{3 k-6}^{k-2}(C)$ such that $\rho\left(L_{C}, y\right)=-1$, then $l_{E}(-(2 k-6) y)$ is a $\mathfrak{g}_{k}^{k-2}$ with vanishing sequence at $y$ being $\geq(0,2,3, \ldots, k-2, k)$. Moreover, at the level of 1 -cycles we have the identification $\sigma^{*}\left(C^{1}\right) \equiv X+\delta(k, 1) T$, where

$$
X:=\left\{(y, D) \in C \times C_{k-2}: h^{0}(C, D+2 y) \geq 2\right\}
$$

and $T$ is the curve consisting of $\mathfrak{g}_{k}^{k-2}$ 's on $E$ with vanishing $\geq(0,2, \ldots, k-2, k)$ at the fixed point $y \in E$.

(2) Let $C_{y}^{0}=C / y \sim q$ be an element of $\Delta_{0}^{0}$. Then limit linear series of type $\mathfrak{g}_{3 k-6}^{k-2}$ on $C_{y}^{0}$ are in 1:1 correspondence with complete linear series $L$ on $C$ of type $\mathfrak{g}_{3 k-6}^{k-2}$ satisfying the condition $h^{0}\left(C, L \otimes \mathcal{O}_{C}(-y-q)\right)=h^{0}(C, L)-1$. Thus there is an isomorphism between the cycle $\sigma^{*}\left(C^{0}\right)$ of $\mathfrak{g}_{3 k-6}^{k-2}$ on all curves $C_{y}^{0}$ with $y \in C$ and the smooth curve

$$
Y:=\left\{(y, D) \in C \times C_{k-2}: h^{0}(C, D+y+q) \geq 2\right\} .
$$

Proof. We only prove (1) the remaining case being similar. Suppose $l=\left(l_{C}, l_{E}\right)$ is a limit $\mathfrak{g}_{3 k-6}^{k-2}$ on $C_{y}^{1}$. Using the additivity of the Brill-Noether number (see e.g. [EH1], Lemma 3.6), we get that $0=\rho(2 k-2, k-2,3 k-6) \geq \rho\left(l_{C}, y\right)+\rho\left(l_{E}, y\right)$. Since the vanishing sequence at $y$ of the $E$-aspect of $l$ is

$$
a^{l_{E}}(y) \leq(2 k-5,2 k-4, \ldots, 3 k-8,3 k-6),
$$

it follows that we also have the inequality $a^{l_{C}}(y) \geq(0,2, \ldots, k-1)$. If $y \in C$ is general, then $\rho\left(l_{C}, y\right)=0$ and $a^{l_{C}}(y)=(0,2, \ldots, k-1)$. Moreover, $l_{C}$ corresponds to a complete linear series $\left|L_{C}\right|$ on $C$ such that $h^{1}\left(C, L_{C}\right)=1$ and $h^{1}\left(L_{C} \otimes \mathcal{O}_{C}(-2 y)\right)=2$. If $y \in C$ is one of the points such that $\rho\left(l_{C}, y\right)=-1$, then since $C$ is Brill-Noether general we must have $a_{0}^{l_{C}}(y)=0$, which implies that $a^{l_{C}}(y)=(0,2, \ldots, k-2, k)$, hence $a^{l_{E}}(y) \geq$ $(2 k-6,2 k-4, \ldots, 3 k-8,3 k-6)$, or equivalently, $l_{E}=(2 k-6) y+\left|V_{E}\right|$, where $V_{E} \subset$ $H^{0}\left(\mathcal{O}_{E}(k y)\right)$ is a $\mathfrak{g}_{k}^{k-2}$ with vanishing sequence $\geq(0,2,3, \ldots, k-2, k)$ at $y$. An easy argument shows that the variety $Y$ of such $V_{E}^{\prime}$ s is isomorphic to $\mathbf{P}\left(\mathcal{O}_{E}(k y)_{\mid 2 y}\right)$.

Proposition 3.4. Let $C_{y}^{j}:=C \cup_{y} D$ be an element from $\Delta_{j}^{0}$, where $2 \leq j \leq k-1, g(C)=$ $2 k-2-j$ and $g(D)=j$. Suppose $\left(l_{C}, l_{D}\right)$ is a limit $\mathfrak{g}_{3 k-6}^{k-2}$ on $C_{y}^{j}$. If $y \in C$ is a general point, then $l_{C}$ has the divisor $(j-2) y$ as base locus and there exists an integer $0 \leq \alpha \leq[j / 2]$ such that $l_{C}(-(j-2) y)=\left|L_{C}\right|$, with $L_{C} \in W_{3 k-j-4}^{k-2}(C)$ satisfying the inequality

$$
a^{L_{C}}(y) \geq(0,1, \ldots, \alpha-1, \alpha+1, \alpha+2, \ldots, j-\alpha, j-\alpha+2, j-\alpha+3, \ldots, k-1, k) .
$$


Moreover $l_{D}$ is one of the $a(j, \alpha)$ linear systems on $D$ of type $(2 k-j-4) y+\left|L_{D}\right|$, with $L_{D} \in W_{k-2+j}^{k-2}(D)$ such that $h^{0}\left(L_{D}(-(k+1-\alpha) y)\right)=\alpha$ and $h^{0}\left(L_{D}(-(k+\alpha-j) y)\right)=j-\alpha$.

If for some integer $0 \leq \alpha \leq[j / 2], y \in C$ is one of the $b(2 k-2-j, k-1+\alpha-j)$ points for which there exists $L_{C} \in \mathrm{Pic}^{3 k-j-3}(C)$ such that $h^{0}\left(L_{C}(-(\alpha+2) y)\right)=k-1-\alpha$ and $h^{0}\left(L_{C}((j-\alpha+2) y)\right)=k-j+\alpha$, then there are two possibilities:

(1) The aspect $l_{C}$ is of the form $(j-2) y+\left|L_{C}(-y)\right|$, whereas $l_{D}=(2 k-j-4) y+\left|L_{D}\right|$, where $L_{D} \in W_{k-2+j}^{k-2}(D)$ satisfies $h^{0}\left(L_{D}(-(k+1-\alpha) y)\right) \geq \alpha$ and $h^{0}\left(L_{D}(-(k+1+\alpha-j) y)\right) \geq$ $j-\alpha-1$.

(2) The aspect $l_{C}$ is of the form $(j-3) y+\left(L_{C}, V_{C}\right)$, where $V_{C} \subset H^{0}\left(L_{C}\right)$ is such that $H^{0}\left(L_{C}(-2 y)\right) \subset V_{C}$, and $l_{D}=(2 k-j-4) y+\left|L_{D}\right|$ with $L_{D} \in W_{k-2+j}^{k-2}(D)$ and

$a^{L_{D}}(y)=(0,1, \ldots, k-1-j+\alpha, k+1-j+\alpha, \ldots, k-1-\alpha, k+1-\alpha, \ldots, k-1, k+1)$.

Finally, if for some $0 \leq \alpha \leq[j / 2], y \in C$ is one of the $\delta(k, j, \alpha)$ points for which there exists $L_{C} \in \operatorname{Pic}^{3 k-4-j}(C)$ such that

$$
a^{L_{C}}(y)=(0,1, \ldots, \alpha-1, \alpha+1, \ldots, j-\alpha, j-\alpha+2, \ldots, k-2, k-1, k+1),
$$

then $l_{C}=(j-2) y+\left|L_{C}\right|$ and $l_{D}=(2 k-j-5) y+l_{D}^{\prime}$, where $l_{D}^{\prime}$ is a (non-complete) $\mathfrak{g}_{k-1+j}^{k-2}$ on $D$ satisfying $a^{l^{\prime}}(y)=(0,2, \ldots, k-1+\alpha-j, k+1+\alpha-j, \ldots, k-\alpha, k+2-\alpha, \ldots, k, k+1)$.

Proof. Since by definition $[D, y] \in \mathcal{M}_{j, 1}$ is Brill-Noether general, we have that $\rho\left(l_{D}, y\right) \geq$ 0 . The locus of those $(C, y) \in \mathcal{M}_{2 k-2-j, 1}$ carrying a $\mathfrak{g}_{3 k-6}^{k-2}$ with $\rho\left(\mathfrak{g}_{3 k-6}^{k-2}, y\right) \leq-2$ is of codimension $\geq 2$, hence we must also have that $\rho\left(l_{C}, y\right) \geq-1$. Using Proposition 1.2 from [EH3] we have that $a_{k-2}^{l_{C}}(y) \leq k-2+j$ and $a_{0}^{l_{C}}(y) \geq j-3$. There are two cases to consider:

(a) If $\rho\left(l_{C}, y\right)=0$, then $\rho\left(l_{D}, y\right)=0$. Assume first that $a_{0}^{l_{C}}(y)=j-2$. Since we have that $0 \leq a_{s}^{l_{C}}(y)-s-(j-2) \leq 2$ for all $0 \leq s \leq k-2$, there must exist an integer $0 \leq \alpha \leq[j / 2]$, such that if $L_{C}$ denotes the $\mathfrak{g}_{3 k-4-j}^{k-2}$ obtained from $l_{C}$ by removing $(j-2) y$, then the vanishing at $y$ is $a^{L_{C}}(y)=(0,1, \ldots, \alpha-1, \alpha+1, \alpha+2, \ldots, j-\alpha, j-\alpha+2, \ldots, k)$. By compatibility, $l_{D}$ has $(2 k-j-4) y$ in its base locus and we write $l_{D}=(2 k-j-4) y+\left|L_{D}\right|$, where $a^{L_{D}}(y)=(0,1, \ldots, k-2+j-\alpha, k+j-\alpha, \ldots, k-1-\alpha, k+1-\alpha, \ldots, k)$. Вy Riemann-Roch, the number of such $L_{D}$ 's equals the number of $\mathfrak{g}_{j-\alpha+1}^{1}$ 's on $D$ with a $(j-2 \alpha+1)$-fold point at $y$ which is computed by $a(j, \alpha)$. If $a_{0}^{l_{C}}(y)=j-3$, we denote by $L_{C} \in \operatorname{Pic}^{3 k-3-j}(C)$ the line bundle obtained from $l_{C}$ by subtracting $(j-3) y$, and again we see that there must exist $0 \leq \alpha \leq[j / 2]$ such that $h^{0}\left(L_{C}(-(j-\alpha+2) y)\right)=k-j+\alpha$ and $h^{0}\left(L_{C}(-(\alpha+2) y)\right)=k-1-\alpha$. By duality, $K_{C} \otimes L_{C}^{\vee} \otimes \mathcal{O}_{C}((j-\alpha+2) y)$ is one of the finitely many $\mathfrak{g}_{k-1-\alpha}^{1}$ 's on $C$ with a $(j-2 \alpha)$-fold point at $y$. This number equals $b(2 k-2-j, k-1+\alpha-j)$ and this explains case (2) of our statement.

(b) If $\rho\left(l_{C}, y\right)=-1$, then $\rho\left(l_{D}, y\right)=1$. An argument similar to that used above gives two possibilities for $a^{l_{C}}(y)$ and the rest follows because $l_{C}$ and $l_{D}$ are compatible at $y$.

Retaining the notation from Proposition 3.4, for each integer $0 \leq \alpha \leq[j / 2]$ we define the following 1-cycles: 


$$
\begin{gathered}
X_{j, \alpha}:=\left\{\left(y, L_{C}\right) \in C \times \operatorname{Pic}^{3 k-j-4}(C): a_{\alpha}^{L_{C}}(y) \geq \alpha+1, a_{j-\alpha}^{L_{C}}(y) \geq j-\alpha+2\right\}, \\
Y_{j, \alpha}^{\prime}:=\left\{(y, l) \in C \times G_{3 k-j-3}^{k-2}(C): a_{1}^{l}(y) \geq 2, a_{\alpha}^{l}(y) \geq \alpha+2, a_{j-\alpha-1}^{l}(y) \geq j-\alpha+2\right\}, \\
Y_{j, \alpha}^{\prime \prime}:=\left\{L_{D} \in \operatorname{Pic}^{k-2+j}(D): a_{k+\alpha-j}^{L_{D}}(y) \geq k+\alpha-j+1, a_{k-\alpha-1}^{L_{D}}(y) \geq k-\alpha+1\right\}
\end{gathered}
$$

and

$Y_{j, \alpha}^{\prime \prime \prime}:=\left\{l \in G_{k-1+j}^{k-2}(D): a_{1}^{l}(y) \geq 2, a_{k-1+\alpha-j}^{l}(y) \geq k+\alpha-j+1, a_{k-1-\alpha}^{l}(y) \geq k-\alpha+2\right\}$.

Via Proposition 3.4 we view $X_{j, \alpha}, Y_{j, \alpha}^{\prime}, Y_{j, \alpha}^{\prime \prime}$ and $Y_{j, \alpha}^{\prime \prime \prime}$ as 1 -cycles on $\widetilde{\mathfrak{G}}_{3 k-6}^{k-2}$. Note that $X_{j, \alpha}$ projects onto $C$ while $Y_{j, \alpha}^{\prime}$ is isomorphic to the union of $b(2 k-2-j, k-1+\alpha-j)$ copies of $\mathbf{P}^{1}$. We can then rephrase Proposition 3.4 as follows:

Proposition 3.5. If $\sigma: \widetilde{\mathfrak{G}}_{3 k-6}^{k-2} \rightarrow \widetilde{\mathcal{M}}_{g}$ is the natural projection and $[C] \in \mathcal{M}_{2 k-2-j}$ where $2 \leq j \leq k-1$, then we have the following numerical equivalence relation between 1-cycles:

$$
\begin{gathered}
\sigma^{*}\left(C^{j}\right) \equiv \sum_{\alpha=0}^{[j / 2]}\left(a(j, \alpha) X_{j, \alpha}+\delta(k, 2 k-2-j, k+\alpha-j) Y_{j, \alpha}^{\prime}+\right. \\
\left.+b(2 k-2-j, k-1+\alpha-j) Y_{j, \alpha}^{\prime \prime}+\delta(j, \alpha) Y_{j, \alpha}^{\prime \prime \prime}\right) .
\end{gathered}
$$

To compute the class of the cycles introduced in Propositions 3.3 and 3.5 , we introduce some notation. Let $C$ be a Brill-Noether general curve of genus $g$. We denote by $C_{d}$ the $d$-th symmetric product of $C$, by $\mathcal{U} \subset C \times C_{d}$ the universal divisor, and by $\mathcal{L}$ the Poincare bundle on $C \times \operatorname{Pic}^{d}(C)$. We also introduce the projections $\pi_{1}: C \times C_{d} \rightarrow C$ and $\pi_{2}: C \times C_{d} \rightarrow C_{d}$ (and we use the same notation for the projections from $C \times \operatorname{Pic}^{d}(C)$ onto the factors). We define the cohomology classes

$$
\eta=\pi_{1}^{*}([\text { point }]) \in H^{2}\left(C \times C_{d}\right) \text { and } x=\pi_{2}^{*}\left(D_{x_{0}}\right) \in H^{2}\left(C \times C_{d}\right),
$$

where $x_{0} \in C$ is an arbitrary point and $D_{x_{0}}:=\left\{D \in C_{d}: x_{0} \in D\right\}$ (the definition of $x$ is obviously independent of the point $\left.x_{0}\right)$. Finally, if $\delta_{1}, \ldots, \delta_{2 g} \in H^{1}(C, \mathbb{Z}) \cong H^{1}\left(C_{d}, \mathbb{Z}\right)$ is a symplectic basis, then we define the class

$$
\gamma:=-\sum_{\alpha=1}^{g}\left(\pi_{1}^{*}\left(\delta_{\alpha}\right) \pi_{2}^{*}\left(\delta_{g+\alpha}\right)-\pi_{1}^{*}\left(\delta_{g+\alpha}\right) \pi_{2}^{*}\left(\delta_{\alpha}\right)\right) .
$$

With these notations we have the formula (cf. [ACGH], p. 338) [U] $\equiv d \eta+\gamma+x$, corresponding to the Hodge decomposition of $[\mathcal{U}]$. We also record the formulas $\gamma^{3}=$ $\gamma \eta=0, \eta^{2}=0$ and $\gamma^{2}=-2 \eta \pi_{2}^{*}(\theta)$, where $\theta \in H^{2}\left(C_{d}, \mathbb{Z}\right)$ is the pullback of the class of the theta divisor on $\operatorname{Jac}(C)$. Similarly, $c_{1}(\mathcal{L})=d \eta+\gamma$, where $\eta, \gamma \in H^{2}\left(C \times \operatorname{Pic}^{d}(C)\right)$ pull back to $\eta, \gamma \in H^{2}\left(C \times C_{d}\right)$. When $d=k-2$ and $g=2 k-3$, we set $\mathcal{M}:=$ $\pi_{1}^{*}\left(K_{C}\right) \otimes \mathcal{O}_{C \times C_{k-2}}(-\mathcal{U})$, hence $c_{1}(\mathcal{M})=(3 k-6) \eta-\gamma-x$.

We now compute the class of the curves $X$ and $Y$ defined in Proposition 3.3 . 
Proposition 3.6. Let $C$ be a Brill-Noether general curve of genus $g=2 k-3$ and $q \in C a$ general point.

(1) The class of the curve $X=\left\{(y, D) \in C \times C_{k-2}: h^{0}(C, D+2 y) \geq 2\right\}$ is given by

$$
[X] \equiv \frac{\theta^{k-2}}{(k-2) !}-\frac{\theta^{k-3} x}{(k-3) !}-\frac{2 \theta^{k-3} \gamma}{(k-3) !}+\frac{2 x \theta^{k-4} \gamma}{(k-4) !}-\frac{4(k+1)}{(k-4) !} x \theta^{k-4} \eta+\frac{4 k-2}{(k-3) !} \theta^{k-3} \eta .
$$

(2) The class of the curve $Y=\left\{(y, D) \in C \times C_{k-2}: h^{0}(C, D+y+q) \geq 2\right\}$ is given by

$$
[Y] \equiv \frac{\theta^{k-2}}{(k-2) !}-\frac{\theta^{k-3} x}{(k-3) !}-\frac{\theta^{k-3} \gamma}{(k-3) !}+\frac{x \theta^{k-4} \gamma}{(k-4) !}-\frac{k+1}{(k-4) !} x \theta^{k-4} \eta+\frac{k-1}{(k-3) !} \theta^{k-3} \eta .
$$

Proof. We will realize both $X$ and $Y$ as pullbacks of degeneracy loci and compute their classes using the Thom-Porteous formula. We consider the map $\epsilon: C \times C_{k-2} \rightarrow C_{k}$ given by $\epsilon(y, D):=2 y+D$. It is easy to check that

$$
\epsilon^{*}(x)=2 \eta+x \text { and } \epsilon^{*}(\theta)=4 g \eta+\theta-2 \gamma .
$$

Then $X=\epsilon^{*}\left(C_{k}^{1}\right)$ and to compute the class of $C_{k}^{1}=\left\{E \in C_{k}: h^{0}(E) \geq 2\right\}$ we introduce the rank $k$ vector bundle $\mathcal{P}:=\left(\pi_{2}\right)_{*}\left(\pi_{1}^{*} K_{C} \otimes \mathcal{O}_{\mathcal{U}}\right)$ on $C_{k}$ having total Chern class $c_{t}(\mathcal{P})=(1-x)^{-k+4} e^{\frac{\theta}{1-x}}$ (cf. [ACGH], pg. 240). There is a natural bundle map $\phi^{\vee}: \epsilon^{*}\left(\mathcal{P}^{\vee}\right) \rightarrow H^{0}\left(K_{C}\right)^{\vee} \otimes \mathcal{O}_{C \times C_{k-2}}$, and $X=Z_{k-1}\left(\phi^{\vee}\right)$. Then by Thom-Porteous, we can write

$$
\begin{aligned}
& {[X]=\left[\frac{1}{c_{t}\left(\epsilon^{*}\left(\mathcal{P}^{\vee}\right)\right)}\right]_{k-2}=\epsilon^{*}\left(\frac{\theta^{k-2}}{(k-2) !}-\frac{x \theta^{k-3}}{(k-3) !}\right)=} \\
& =\frac{(4 g \eta+\theta-2 \gamma)^{k-2}}{(k-2) !}-\frac{(4 g \eta+\theta-2 \gamma)^{k-3}(2 \eta+x)}{(k-3) !},
\end{aligned}
$$

which quickly leads to the desired expression for $[X]$.

To compute the class of $Y$ we proceed in a similar manner: we consider the map $\chi: C \times C_{k-2} \rightarrow C_{k}$ given by $\chi(y, D):=y+q+D$. Then $\chi^{*}(x)=x+\eta$ and $\chi^{*}(\theta)=$ $g \eta+\theta-\gamma$. For each $(y, D) \in C \times C_{k-2}$ we have a natural map

$$
H^{0}\left(K_{C \mid y+q+D}\right)^{\vee} \rightarrow H^{0}\left(K_{C}\right)^{\vee}
$$

which globalizes to a vector bundle map $\psi^{\vee}: \chi^{*}\left(\mathcal{P}^{\vee}\right) \rightarrow H^{0}\left(K_{C}\right)^{\vee} \otimes \mathcal{O}_{C \times C_{k-2}}$ and then it is clear that $Y=Z_{k-1}\left(\psi^{\vee}\right)$. Applying Thom-Porteous again, we obtain

$$
\begin{aligned}
{[Y] } & =\left[\frac{1}{c_{t}\left(\chi^{*}\left(\mathcal{P}^{\vee}\right)\right)}\right]_{k-2}=\chi^{*}\left(\frac{\theta^{k-2}}{(k-2) !}-\frac{x \theta^{k-3}}{(k-3) !}\right)= \\
& =\frac{(g \eta+\theta-\gamma)^{k-2}}{(k-2) !}-\frac{(g \eta+\theta-\gamma)^{k-3}(\eta+x)}{(k-3) !},
\end{aligned}
$$

which after a straightforward calculation gives the class of $Y$.

We also need the following intersection theoretic result:

Lemma 3.7. For $j \geq 1$ we denote by $\mathcal{F}_{j}:=\left(\pi_{2}\right)_{*}\left(\mathcal{M}^{\otimes j}\right)$ the vector bundle on $C_{k-2}$ with fibre $\mathcal{F}_{j}(D)=H^{0}\left(C, j K_{C}-j D\right)$ over each $D \in C_{k-2}$. Then $c_{1}\left(\mathcal{F}_{j}\right)=-j^{2} \theta-j(3 j-2)(k-2) x$. 
Proof. We apply Grothendieck-Riemann-Roch for the map $\pi_{2}: C \times C_{k-2} \rightarrow C_{k-2}$. We obtain that

$$
\begin{gathered}
c_{1}\left(\mathcal{F}_{j}\right)=\left[\left(\pi_{2}\right)_{*}\left(\left(1+j((3 k-6) \eta-\gamma-x)+\frac{j^{2}}{2}((3 k-6) \eta-\gamma-x)^{2}\right) \cdot(1-(2 k-4) \eta)\right)\right]_{1}= \\
=-j^{2} \theta-j(3 j-2)(k-2) x
\end{gathered}
$$

Next we compute the class of $X_{j, \alpha}$ when $2 \leq j \leq k-1$. It is convenient to state our result as follows:

Proposition 3.8. Let $C$ be a general curve of genus $2 k-2-j$ such that $2 \leq j \leq k-1$. We have the following relation in $H^{4 k-4-2 j}\left(C \times \mathrm{Pic}^{3 k-j-4}(C)\right)$ :

$$
\begin{aligned}
& \sum_{\alpha=0}^{[j / 2]} a(j, \alpha) X_{j, \alpha} \equiv \frac{2(2 k-3) !}{k !(k-2) !(2 k-3-j) !}\left(\frac{\theta^{2 k-2-j}}{2 k-2-j}+\frac{(3 k j+12 k-9 j-18) \theta^{2 k-3-j} \gamma}{2 k-3}+\right. \\
& \left.\frac{\theta^{2 k-3-j} \eta}{(2 k-5)(2 k-3)}\left(60-64 k+16 k^{2}+j\left(60-\frac{67}{2} k-10 k^{2}+6 k^{3}\right)+j^{2}\left(15+2 k-5 k^{2}\right)+\frac{3}{2} k j^{3}\right)\right) .
\end{aligned}
$$

Proof. We fix an integer $0 \leq \alpha \leq[j / 2]$ and a divisor $D \in C_{e}$ of degree $e \geq k-j-1$. We set $d:=3 k-4-j, \Gamma:=D \times \operatorname{Pic}^{d}(C)$ and denote by $u, v: C \times C \times \operatorname{Pic}^{d}(C) \rightarrow C \times \operatorname{Pic}^{d}(C)$ the two projections and by $J_{\alpha}(\mathcal{L})$ the $\alpha$-th jet bundle of the Poincare bundle on $C \times \operatorname{Pic}^{d}(C)$. Then $\mathcal{P}(D):=\left(\pi_{2}\right)_{*}\left(\mathcal{L} \otimes \mathcal{O}_{C \times \operatorname{Pic}^{d}(C)}(\Gamma)\right)$ is a vector bundle of rank $e+k-1$. For each $\alpha \geq 0$ we define the vector bundle $J_{\alpha}(\mathcal{L}, D):=u_{*}\left(v^{*}(\mathcal{L}) \otimes \mathcal{O}_{(\alpha+1) \Delta+v^{*}(\Gamma)}\right)$, where $\Delta \subset C \times C \times \operatorname{Pic}^{d}(C)$ is the diagonal, and consider the vector bundle map

$$
\phi_{\alpha}: J_{\alpha}(\mathcal{L}, D)^{\vee} \rightarrow\left(\pi_{2}\right)^{*}(\mathcal{P}(D))^{\vee}
$$

which on fibres is the dual of the map $H^{0}\left(L_{C} \otimes \mathcal{O}(D)\right) \rightarrow H^{0}\left(L_{C} \otimes \mathcal{O}_{(\alpha+1) y+D}(D)\right)$ for each pair $\left(y, L_{C}\right) \in C \times \operatorname{Pic}^{d}(C)$.

The cycle $X_{j, \alpha}$ consists of pairs $\left(y, L_{C}\right)$ such that $\operatorname{dim} \operatorname{Ker}\left(\phi_{\alpha}\left(y, L_{C}\right)\right) \geq 1$ and $\operatorname{dim} \operatorname{Ker}\left(\phi_{j-\alpha+1}\left(y, L_{C}\right)\right) \geq 2$ and its class is given by the formula (cf. [Fu],Theorem 14.3):

$$
X_{j, \alpha} \equiv c_{j+1-\alpha}(\alpha) c_{k-1+\alpha-j}(j-\alpha+1)-c_{k-\alpha}(\alpha) c_{k-2+\alpha-j}(j-\alpha+1),
$$

where $c_{l}(\beta):=c_{l}\left(\left(\pi_{2}\right)^{*}(\mathcal{P}(D))^{\vee}-J_{\beta}(\mathcal{L}, D)^{\vee}\right)$. Since $c_{\text {tot }}\left(\left(\pi_{2}\right)^{*}(\mathcal{P}(D))=e^{\theta}\right.$ and $c_{1}(\mathcal{L})=$ $d \eta+\gamma$ (independent of $D$ !), after a short calculation we obtain that

$c_{l}(\beta)=\frac{\theta^{l}}{l !}+\frac{\theta^{l-1}}{(l-1) !}((\beta+1) \gamma+(\beta+1)(d+\beta(2 k-3-j)) \eta)-\frac{\theta^{l-1}}{(l-2) !}(\beta+1)(\beta+2) \eta$.

A straightforward computation now leads to the stated formula.

For each integers $0 \leq a \leq k-2$ and $b \geq 2$ we shall define vector bundles $\mathcal{G}_{a, b}$ over $\widetilde{\mathfrak{G}}_{3 k-6}^{k-2}$ with fibre

$$
\mathcal{G}_{a, b}(C, L)=H^{0}\left(\wedge^{a} M_{L} \otimes L^{\otimes b}\right)
$$


over every point $(C, L) \in \mathfrak{G}_{3 k-6}^{k-2}$. Note that $\mathcal{G}_{i, 2 \mid \mathfrak{G}_{3 k-6}^{k-2}}=\mathcal{B}$, where $\mathcal{B}$ is the vector bundle we introduced in Proposition 2.6 Of course, the question is how to extend this description over the divisors $\Delta_{j}^{0}$. First we will extend $\mathcal{G}_{a, b}$ over $\sigma^{-1}\left(\mathcal{M}_{g}^{0} \cup \Delta_{0}^{0} \cup \Delta_{1}^{0}\right)$ and we start by constructing the vector bundles $\mathcal{G}_{0, b}$ :

Proposition 3.9. For each $b \geq 2$ there exists a vector bundle $\mathcal{G}_{0, b}$ over $\sigma^{-1}\left(\mathcal{M}_{g}^{0} \cup \Delta_{1}^{0} \cup \Delta_{0}^{0}\right) \subset$ $\widetilde{\mathfrak{G}}_{3 k-6}^{k-2}$ of rank $k(3 b-2)-6 b+3$ whose fibres admit the following description:

- For $(C, L) \in \mathfrak{G}_{3 k-6}^{k-2}$, we have that $\mathcal{G}_{0, b}(C, L)=H^{0}\left(C, L^{\otimes b}\right)$.

- For $t=\left(C \cup_{y} E, L\right) \in \sigma^{-1}\left(\Delta_{1}^{0}\right)$, where $L$ is the linear series $\mathfrak{g}_{3 k-6}^{k-2}$ on $C$ determining a limit $\mathfrak{g}_{3 k-6}^{k-2}$ on $C \cup_{y} E$, we have that

$$
\mathcal{G}_{0, b}(t)=H^{0}\left(C, L^{\otimes b}(-2 y)\right)+\mathbb{C} \cdot u^{b} \subset H^{0}\left(C, L^{\otimes b}\right),
$$

where $u \in H^{0}(C, L)$ is any section such that $\operatorname{ord}_{y}(u)=0$.

- For $t=(C / y \sim q, L) \in \sigma^{-1}\left(\Delta_{0}^{0}\right)$, where $q, y \in C$ and $L$ is $a \mathfrak{g}_{3 k-6}^{k-2}$ on $C$, we have that

$$
\mathcal{G}_{0, b}(t)=H^{0}\left(C, L^{\otimes b}(-y-q)\right) \oplus \mathbb{C} \cdot u^{b} \subset H^{0}\left(C, L^{\otimes b}\right),
$$

where $u \in H^{0}(C, L)$ is a section such that $\operatorname{ord}_{y}(u)=\operatorname{ord}_{q}(u)=0$.

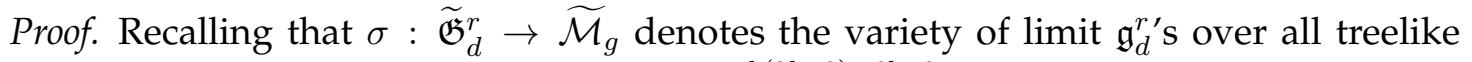
curves of genus $g$, the morphism $\mathfrak{G}_{3 k-6}^{k-2} \rightarrow \mathfrak{G}_{b(3 k-6)}^{k(3 b-2)-6 b+2}$ given by $(C, L) \mapsto\left(C, L^{\otimes b}\right)$ can be extended to a morphism $\nu_{b}: \widetilde{\mathfrak{G}}_{3 k-6}^{k-2} \rightarrow \widetilde{\mathfrak{G}}_{b(3 k-6)}^{k(3 b-2)-6 b+2}$ over $\mathcal{M}_{g} \cup \Delta_{0}^{0} \cup \Delta_{1}^{0}$ as follows. We fix a point $t=\left(C \cup_{y} E, l=\left(l_{C}, l_{E}\right)\right)$ a limit $\mathfrak{g}_{3 k-6}^{k-2}$, where $l_{C}=\left(L_{C}, H^{0}\left(L_{C}\right)\right) \in G_{3 k-6}^{k-2}(C)$ is such that $H^{0}\left(L_{C}\right)=H^{0}\left(L_{C}(-2 y)\right) \oplus \mathbb{C} \cdot u$, for a certain $u \in H^{0}\left(L_{C}\right)$ with $\operatorname{ord}_{y}(u)=0$, while $l_{E}=\left(\mathcal{O}_{E}((3 k-6) y),(2 k-6) p+\left|V_{E}\right|\right)$ (any limit $\mathfrak{g}_{3 k-6}^{k-2}$ on $C \cup_{y} E$ is of this form). Then we set $\nu_{b}(t):=\left(l_{C}^{b}, l_{E}^{b}\right)$, where $l_{C}^{b}=\left(L_{C}^{\otimes b}, H^{0}\left(L_{C}^{\otimes b}(-2 y)\right) \oplus \mathbb{C} \cdot u^{b} \subset H^{0}\left(L_{C}^{\otimes b}\right)\right)$ and $l_{E}^{b}=\left(\mathcal{O}_{E}(b(3 k-6) p),(2 k-3) p+|(k(3 b-2)-6 b+3) p|\right)$. In other words, $\left(l_{C}^{b}, l_{E}^{b}\right)$ is the $b$-th power of the original limit linear series $\mathfrak{g}_{3 k-6}^{k-2}$.

For a point $z=\left(C / y \sim q, L_{C}\right) \in \Delta_{0}^{0}$ with $L_{C}$ being a $\mathfrak{g}_{3 k-6}^{k-2}$ such that $H^{0}\left(L_{C}\right)=$ $H^{0}\left(L_{C}(-y-q)\right) \oplus \mathbb{C} \cdot u$, we define $\nu_{b}(z):=\left(L_{C}^{\otimes b}, H^{0}\left(L_{C}^{\otimes b}(-y-q)\right) \oplus \mathbb{C} \cdot u^{b} \subset H^{0}\left(L_{C}^{\otimes b}\right)\right)$. The fact that $\nu_{b}$ can be constructed algebraically follows easily from the equations of the scheme $\widetilde{\mathfrak{G}}_{3 k-6}^{k-2}$ described in [EH1], pg. 358. The variety $\widetilde{\mathfrak{G}}_{b(3 k-6)}^{k(3 b-2)-6 b+2}$ carries a tautological vector bundle $\mathcal{T}=\mathcal{T}_{C}$ with fibre over each point corresponding to a curve from $\mathcal{M}_{g}^{0} \cup \Delta_{0}^{0}$ being the space of global sections of the linear series, while the fibre over a point corresponding to a curve from $\Delta_{1}^{0}$ is the space of sections of the aspect of the limit linear series corresponding to the curve of genus $g-1$. We define $\mathcal{G}_{0, b}:=\nu_{b}^{*}(\mathcal{T})$. The description of the fibres of the vector bundle $\mathcal{G}_{0, b}$ is now immediate.

Having defined the vector bundles $\mathcal{G}_{0, b}$ we now define inductively all vector bundles $\mathcal{G}_{a, b}$ : first we define $\mathcal{G}_{1, b}$ as the kernel of the multiplication map $\mathcal{G}_{0,1} \otimes \mathcal{G}_{0, b} \rightarrow \mathcal{G}_{0, b+1}$, that is, by the exact sequence

$$
0 \longrightarrow \mathcal{G}_{1, b} \longrightarrow \mathcal{G}_{0,1} \otimes \mathcal{G}_{0, b} \longrightarrow \mathcal{G}_{0, b+1} \longrightarrow 0 .
$$


Having defined $\mathcal{G}_{l, b}$ for all $l \leq a-1$, we define the vector bundle $\mathcal{G}_{a, b}$ by the exact sequence

$$
0 \longrightarrow \mathcal{G}_{a, b} \longrightarrow \wedge^{a} \mathcal{G}_{0,1} \otimes \mathcal{G}_{0, b} \stackrel{d_{a, b}}{\longrightarrow} \mathcal{G}_{a-1, b+1} \longrightarrow 0 .
$$

Proposition 3.10. For integers $b \geq 2$ and $1 \leq a \leq i$, the Koszul multiplication map $d_{a, b}$ : $\wedge^{a} \mathcal{G}_{0,1} \otimes \mathcal{G}_{0, b} \rightarrow \mathcal{G}_{a-1, b+1}$ is well-defined and surjective. In particular one can define the vector bundle $\mathcal{G}_{a, b}:=\operatorname{Ker}\left(d_{a, b}\right)$ over $\sigma^{-1}\left(\mathcal{M}_{g}^{0} \cup \Delta_{0}^{0} \cup \Delta_{1}^{0}\right)$ and the sequence (4) makes sense.

Proof. We do induction on $a$ and start with the case $a=1$. We only check the surjectivity of the map $\mathcal{G}_{0,1} \otimes \mathcal{G}_{0, b} \rightarrow \mathcal{G}_{0, b+1}$ over the locus $\sigma^{-1}\left(\Delta_{1}^{0}\right)$, the other cases, namely $\sigma^{-1}\left(\mathcal{M}_{g}^{0}\right)$ and $\sigma^{-1}\left(\Delta_{0}^{0}\right)$ being quite similar. It is enough to show that if $C$ is a sufficiently general curve of genus $2 k-3, y \in C$ is a point and $L \in W_{3 k-6}^{k-2}(C)$ is a linear system with a cusp at $y$, then the map $H^{0}(L) \otimes H^{0}\left(L^{\otimes b}(-2 y)\right) \rightarrow H^{0}\left(L^{\otimes(b+1)}(-2 y)\right)$ is onto. This is equivalent to $H^{1}\left(M_{L} \otimes L^{\otimes b}(-2 y)\right)=0$ which follows because $M_{L}$ is stable (cf. Lemma 2.11.

We now treat the general case $1 \leq a \leq i$ and we want to show that the Koszul map $d_{a, b}: \wedge^{a} H^{0}(L) \otimes \mathcal{G}_{0, b}(L) \rightarrow \mathcal{G}_{a-1, b+1}(L)$ is surjective for each $b \geq 2$ and for each $(C, L)$ as above. For simplicity we have denoted $\mathcal{G}_{a, b}(L)=\mathcal{G}_{a, b}(t)$, where $t \in \sigma^{-1}\left(\Delta_{1}^{0}\right)$ is the point $\left(C \cup_{y} E, l\right), E$ is an arbitrary elliptic tail and $l$ is a limit linear series having $L$ as its $C$-aspect. We can then write $H^{0}(L) / H^{0}(L(-2 y))=\mathbb{C} \cdot u$, where $u \in H^{0}(L)$ is uniquely determined up to translation by an element from $H^{0}(L(-2 y))$. We first claim that the restriction of the Koszul differential

$$
d_{a, b}^{0}: \wedge^{a} H^{0}(L) \otimes H^{0}\left(L^{\otimes b}(-2 y)\right) \rightarrow H^{0}\left(\wedge^{a-1} M_{L} \otimes L^{\otimes(b+1)}(-2 y)\right)
$$

is surjective. This is so because $\operatorname{Coker}\left(d_{a, b}^{0}\right)=H^{1}\left(\wedge^{a} M_{L} \otimes L^{\otimes b}(-2 y)\right)=0$ (use again that $M_{L}$ is a stable bundle). But then since

$$
H^{0}\left(\wedge^{a-1} M_{L} \otimes L^{\otimes(b+1)}(-2 y)\right) \subset \mathcal{G}_{a-1, b+1}(L)
$$

is a linear subspace of codimension $\left(\begin{array}{l}k-2 \\ a-1\end{array}\right)$, to prove that $d_{a, b}$ itself is surjective it suffices to notice that the image of the injective map $\wedge^{a-1} H^{0}(L(-2 y)) \rightarrow \wedge^{a-1} H^{0}(L) \otimes \mathcal{G}_{0, b+1}(L)$ given by

$f_{1} \wedge \ldots \wedge f_{a-1} \mapsto f_{1} \wedge \ldots \wedge f_{a-1} \otimes u^{b+1}-\sum_{l=1}^{a-1}(-1)^{l-1} u \wedge f_{1} \wedge \ldots \wedge \check{f}_{l} \wedge \ldots \wedge f_{a-1} \otimes f_{l} u^{b}$

is entirely contained in $\operatorname{Im}\left(d_{a, b}\right)$ and is clearly disjoint from $H^{0}\left(\wedge^{a-1} M_{L} \otimes L^{\otimes(b+1)}(-2 y)\right)$.

To compute the intersection numbers of the divisors $\overline{\mathcal{Z}}_{g, i}$ with the test curves $C^{0}$ and $C^{1}$ we need to understand the restriction of the vector bundle $\mathcal{G}_{i, 2}$ to $X$ and $Y$.

Proposition 3.11. Let $C$ be a general curve of genus $2 k-3$ and $k=3 i+6$ with $i \geq 0$. If $C^{1}$ is the test curve in $\Delta_{1}$ obtained by attaching to $C$ a fixed elliptic tail at a varying point of $C$ and $X=\sigma^{-1}\left(C^{1}\right)$, then we have the following formula:

$$
c_{1}\left(\mathcal{G}_{2, i \mid X}\right)=\left(\begin{array}{c}
3 i+4 \\
i
\end{array}\right)\left(c_{x} x+c_{\eta} \eta+c_{\gamma} \gamma+c_{\theta} \theta\right)
$$


where

$$
\begin{gathered}
c_{\eta}=-27 i+40, c_{x}=-\frac{27 i^{4}+153 i^{3}+331 i^{2}+323 i+120}{(3 i+4)(i+1)} \\
c_{\gamma}=\frac{5 i+8}{3 i+4}, c_{\theta}=-\frac{27 i^{3}+101 i^{2}+124 i+48}{(3 i+3)(3 i+4)} .
\end{gathered}
$$

Proof. For $j \geq 2$ we define the jet bundle $J_{1}\left(\mathcal{M}^{\otimes j}\right):=\left(u_{*}\right)\left(v^{*}\left(\mathcal{O}_{C \times C_{k-2}}\left(\mathcal{M}^{\otimes j}\right)\right) \otimes \mathcal{O}_{2 \Delta}\right)$, where $u, v: C \times C \times C_{k-2} \rightarrow C \times C_{k-2}$ are the projections. We have two exact sequences of vector bundles on $X$ :

$$
0 \longrightarrow u_{*}\left(v^{*}\left(\mathcal{M}^{\otimes j}\right) \otimes \mathcal{O}(-2 \Delta)\right)_{\mid X} \longrightarrow \mathcal{G}_{0, j \mid X} \longrightarrow \mathcal{M}_{\mid X}^{\otimes j} \longrightarrow 0
$$

and

$$
0 \longrightarrow u_{*}\left(v^{*}\left(\mathcal{M}^{\otimes j}\right) \otimes \mathcal{O}(-2 \Delta)\right)_{\mid X} \longrightarrow \pi_{2}^{*}\left(\mathcal{F}_{j}\right)_{\mid X} \longrightarrow J_{1}\left(\mathcal{M}^{\otimes j}\right)_{\mid X} \longrightarrow 0
$$

from which we can write that

$$
\begin{gathered}
c_{1}\left(\mathcal{G}_{0, j \mid X}\right)=c_{1}\left(\pi_{2}^{*}\left(\mathcal{F}_{j}\right)_{\mid X}\right)-c_{1}\left(\left(\pi_{1}^{*} K_{C} \otimes \mathcal{M}^{\otimes j}\right)_{\mid X}\right)= \\
=-j^{2} \theta-(3 j+4)(k-2) \eta+j \gamma-j((3 j-2)(k-2)-1) x .
\end{gathered}
$$

From the exact sequences defining $\mathcal{G}_{i, 2}$ we then obtain that

$$
c_{1}\left(\mathcal{G}_{i, 2 \mid X}\right)=\sum_{l=0}^{i}(-1)^{l} c_{1}\left(\wedge^{i-l} \mathcal{G}_{0,1 \mid X} \otimes \mathcal{G}_{0, l+2 \mid X}\right) .
$$

Note that Riemann-Roch gives that $\operatorname{rk}\left(\mathcal{G}_{0, l+2}\right)=(3 k-6) l+4 k-9$, while $c_{1}\left(\wedge^{i-l} \mathcal{G}_{0,1 \mid X}\right)=$ $\left(\begin{array}{c}k-2 \\ i-l-1\end{array}\right)(-\theta-(k-2) x)$. To obtain a closed formula for $c_{1}\left(\mathcal{G}_{i, 2 \mid X}\right)$ we now specialize to the case $k=3 i+6$ and we write that

$$
\begin{gathered}
c_{1}\left(\mathcal{G}_{i, 2 \mid X}\right)=\sum_{l=0}^{i}(-1)^{l}\left[((9 i+12) l+(12 i+15))\left(\begin{array}{c}
3 i+4 \\
i-l-1
\end{array}\right)(-\theta-(3 i+4) x)+\right. \\
\left.+\left(\begin{array}{c}
3 i+5 \\
i-l
\end{array}\right)\left(-(l+2)^{2} \theta+(l+2) \gamma-(3 i+4)(3 l+10) \eta-(l+2)((3 l+4)(3 i+4)-1) x\right)\right] .
\end{gathered}
$$

A long but elementary calculation leads to the stated formula.

A similar calculation yields the first Chern class of $\mathcal{G}_{i, 2 \mid Y}$ :

Proposition 3.12. Let $(C, q)$ be a general pointed curve of genus $2 k-3$ and $C^{0}$ the test curve in $\Delta_{0}$ obtained by identifying the fixed point $q$ with a varying point $y$ on $C$. If $Y=\sigma^{-1}\left(C^{0}\right)$, then we have the formula

$$
c_{1}\left(\mathcal{G}_{i, 2 \mid Y}\right)=\left(\begin{array}{c}
3 i+4 \\
i
\end{array}\right)\left(d_{x} x+\eta+d_{\theta} \theta\right)
$$

where

$$
d_{x}=-\frac{27 i^{4}+153 i^{3}+331 i^{2}+323 i+120}{(3 i+4)(i+1)}, d_{\theta}=-\frac{27 i^{3}+101 i^{2}+124 i+48}{(3 i+3)(3 i+4)} .
$$


Proof. We define $\Gamma_{q}:=C \times\{q\} \times C_{k-2}$ and denote by $\iota: C \times C_{k-2} \hookrightarrow \Gamma_{q}$ the inclusion map. Then we have the exact sequences on $Y$

$$
0 \longrightarrow\left(u_{*}\right)\left(v^{*}\left(\mathcal{M}^{\otimes j}\right) \otimes \mathcal{O}\left(-\Delta-\Gamma_{q}\right)\right)_{\mid Y} \longrightarrow \mathcal{G}_{0, j \mid Y} \longrightarrow \mathcal{M}_{\mid Y}^{\otimes j} \longrightarrow 0
$$

and

$$
0 \longrightarrow \iota^{*}\left(v^{*}\left(\mathcal{M}^{\otimes j}\right) \otimes \mathcal{O}(-\Delta)\right)_{\mid Y} \longrightarrow\left(u_{*}\right)\left(v^{*}\left(\mathcal{M}^{\otimes j}\right) \otimes \mathcal{O}_{\Delta+\Gamma_{q}}\right)_{\mid Y} \longrightarrow \pi_{2}^{*}\left(\mathcal{F}_{j}\right)_{\mid Y} \longrightarrow 0 .
$$

Using that $c_{1}(\mathcal{M})=(3 k-6) \eta-\gamma-x$, it is now straightforward to check that

$$
c_{1}\left(\iota^{*}\left(v^{*}\left(\mathcal{M}^{\otimes j}\right) \otimes \mathcal{O}(-\Delta)\right)\right)=-j x-\eta,
$$

hence $c_{1}\left(\mathcal{G}_{0, j \mid Y}\right)=c_{1}\left(\pi_{2}^{*}\left(\mathcal{F}_{j}\right)\right)_{\mid Y}+j x+\eta=-j^{2} \theta+\eta-j((3 j-2)(k-2)-1) x$. But then as in the previous proposition we have that

$$
c_{1}\left(\mathcal{G}_{i, 2 \mid Y}\right)=\sum_{l=0}^{i}(-1)^{l} c_{1}\left(\wedge^{i-l} \mathcal{G}_{0,1 \mid Y} \otimes \mathcal{G}_{0, l+2 \mid Y}\right),
$$

which after some calculations yields the stated formula in the special case $k=3 i+6$.

In what follows we extend the vector bundles $\mathcal{G}_{a, b}$ over the whole $\widetilde{\mathfrak{G}}_{3 k-6}^{k-2}$. Roughly speaking, the fibre of $\mathcal{G}_{a, b}$ over a point corresponding to a singular curve $C \cup_{y} D$ will be the space $H^{0}\left(C \cup D, \wedge^{a} M_{L} \otimes L^{\otimes b}\right)$, where $L$ will be a line bundle on $C \cup D$ obtained by twisting appropriately a limit $\mathfrak{g}_{3 k-6}^{k-2}$ on $C \cup D$. The twisting is chosen such that, the vector bundles $\mathcal{G}_{a, b}$ sit in the exact sequences (4). In the next statement we use the notation introduced in Proposition 3.5

Theorem 3.13. For integers $0 \leq a \leq i, b \geq 2$, or $(a, b)=(0,1)$, there exists a vector bundle $\mathcal{G}_{a, b}$ over $\widetilde{\mathfrak{G}}_{3 k-6}^{k-2}$ having the following properties:

(1) If $(C, L) \in \mathfrak{G}_{3 k-6}^{k-2}$, then $\mathcal{G}_{a, b}(C, L)=H^{0}\left(\wedge^{a} M_{L} \otimes L^{\otimes b}\right)$, that is, $\mathcal{G}_{a, b \mid \mathfrak{G}_{3 k-6}^{k-2}}=\mathcal{G}_{a, b}$.

$$
\text { If } t=\left(C \cup_{y} D, L_{C}, L_{D}\right) \in \sigma^{-1}\left(\Delta_{j}^{0}\right) \subset \widetilde{\mathfrak{G}}_{3 k-6}^{k-2} \text {, with } 2 \leq g(D)=j \leq k-1, g(C)=
$$
$2 k-2-j, L_{C} \in W_{3 k-4-j}^{k-2}(C)$ and $L_{D} \in W_{k-2+j}^{k-2}(D)$, then there are two situations:

(2) If $2 \leq j \leq 2 i+2$, then $\mathcal{G}_{a, b}(t)=H^{0}\left(C \cup D, \wedge^{a} M_{L_{C \cup D}} \otimes L_{C \cup D}^{\otimes b}\right)$, where $L_{C \cup D}=$ $\left(L_{C}(-(j+2) y), L_{D}(-(k-2-j) y)\right) \in \mathrm{Pic}^{3 k-6-2 j}(C) \times \mathrm{Pic}^{2 j}(D)$ is a globally generated line bundle on $C \cup D$.

(3) If $2 i+3 \leq j \leq 3 i+5$ and $c:=[(j+2) / 2]$, then $\mathcal{G}_{a, b}(t)=H^{0}\left(C \cup D, \wedge^{a} M_{L_{C \cup D}} \otimes L_{C \cup D}^{\otimes b}\right)$, where $\left.L_{C \cup D}=\left(L_{C}(-c y)\right), L_{D}(-(k-c) y)\right) \in \mathrm{Pic}^{3 k-4-j-c}(C) \times \mathrm{Pic}^{j+c-2}(D)$ is a globally generated line bundle on $C \cup D$.

(4) There are exact sequences over $\widetilde{\mathfrak{G}}_{3 k-6}^{k-2}: 0 \longrightarrow \mathcal{G}_{a, b} \longrightarrow \wedge^{a} \mathcal{G}^{0,1} \otimes \mathcal{G}_{0, b} \longrightarrow \mathcal{G}_{a-1, b+1} \longrightarrow 0$.

Proof. For $j \geq 2$ the divisors $\Delta_{j}^{0}$ are mutually disjoint and we can carry out the construction of $\mathcal{G}_{a, b}$ over each open set $\sigma^{-1}\left(\mathcal{M}_{g}^{0} \cup \Delta_{j}^{0}\right) \subset \widetilde{\mathfrak{G}}_{3 k-6}^{k-2}$ and glue the resulting bundles together. We denote by $\pi: \mathcal{C}_{j} \rightarrow \mathcal{M}_{g}^{0} \cup \Delta_{j}^{0}$ the restriction of the universal curve and by $p: \mathcal{Y}=\mathcal{C}_{j} \times_{\mathcal{M}_{g}^{0} \cup \Delta_{j}^{0}} \widetilde{\mathfrak{G}}_{3 k-6}^{k-2} \rightarrow \widetilde{\mathfrak{G}}_{3 k-6}^{k-2}$. Then $(\sigma p)^{-1}\left(\Delta_{j}^{0}\right)=D_{j}+D_{2 k-2-j}$, where $D_{j}$ (resp. $D_{2 k-2-j}$ ) denotes the divisor in $\mathcal{Y}$ corresponding to the marked point being 
on the genus $j$ (resp. $2 k-2-j$ ) component. If $\mathcal{L}=\mathcal{L}_{0,3 k-6}$ is the Poincare bundle on $\mathcal{Y}$ chosen such that it parametrizes bundles having bidegree $(0,3 k-6)$ on curves $\left[D \cup_{y} C\right] \in \Delta_{j}^{0}$, then for $b \geq 1$ we set

$$
\mathcal{G}_{0, b}:=p_{*}\left(\mathcal{L}^{\otimes b} \otimes \mathcal{O}_{\mathcal{Y}}\left(-2 b j D_{j}\right)\right), \text { when } 2 \leq j \leq 2 i+2,
$$

and $\mathcal{G}_{0, b}:=p_{*}\left(\mathcal{L}^{\otimes b} \otimes \mathcal{O}_{\mathcal{Y}}\left(-b(j-2+c) D_{j}\right)\right)$ when $2 i+3 \leq j \leq 3 i+5$. For each point $t=\left(R=C \cup_{y} D, L_{C}, L_{D}\right) \in \sigma^{-1}\left(\Delta_{j}^{0}\right)$, we have that $\mathcal{G}_{0, b}(t)=H^{0}\left(R, L_{R}^{\otimes b}\right)$, where in the case $j \leq 2 i+2$ we have $L_{R}=\left(L_{C}(-(j+2) y), L_{D}(-(k-2-j) y)\right) \in \operatorname{Pic}^{3 k-6}(R)$ and since $H^{1}\left(R, L_{R}^{\otimes b}\right)=0$ for $b \geq 2$, it follows that $\mathcal{G}_{0, b}$ is a vector bundle for all $b \geq 1$. When $1 \leq a \leq i$, the sheaves $\mathcal{G}_{a, b}$ are defined inductively using the sequences (4). For this to make sense and in order to conclude that $\mathcal{G}_{a, b}$ are vector bundles, one has to show that

$$
H^{1}\left(R, \wedge^{a} M_{L_{R}} \otimes L_{R}^{\otimes b}\right)=0,
$$

for all $b \geq 2$ and $0 \leq a \leq i$. To achieve this we use the Mayer-Vietoris sequence on $R$ :

$0 \rightarrow \wedge^{a} M_{L_{R}} \otimes L_{R}^{\otimes b} \longrightarrow\left(\wedge^{a} M_{L_{R}} \otimes L_{R}^{\otimes b}\right)_{\mid C} \oplus\left(\wedge^{a} M_{L_{R}} \otimes L_{R}^{\otimes b}\right)_{\mid D} \longrightarrow \wedge^{a} M_{L_{R}} \otimes L_{R}^{\otimes b} \otimes \mathbb{C}(y) \rightarrow 0$,

together with the exact sequences

$$
0 \longrightarrow H^{0}\left(L_{R \mid C}(-y)\right) \otimes \mathcal{O}_{D} \longrightarrow M_{L_{R}} \otimes \mathcal{O}_{D} \longrightarrow M_{L_{R \mid D}} \longrightarrow 0,
$$

and

$$
0 \longrightarrow H^{0}\left(L_{R \mid D}(-y)\right) \otimes \mathcal{O}_{C} \longrightarrow M_{L_{R}} \otimes \mathcal{O}_{C} \longrightarrow M_{L_{R \mid C}} \longrightarrow 0 .
$$

We obtain that (5) holds if we can show that

$$
H^{1}\left(C, \wedge^{a} M_{L_{R \mid C}} \otimes L_{R \mid C}^{\otimes b}\right)=H^{1}\left(C, \wedge^{a} M_{L_{R \mid C}} \otimes L_{R \mid C}^{\otimes b}(-y)\right)=0, \text { and }
$$

and

$$
H^{1}\left(D, \wedge^{a} M_{L_{R \mid D}} \otimes L_{R \mid D}^{\otimes b}\right)=H^{1}\left(D, \wedge^{a} M_{L_{R \mid D}} \otimes L_{R \mid D}^{\otimes b}(-y)\right)=0 .
$$

We only check this when $j \leq 2 i+2$ the remaining case being similar. Since $h^{0}\left(L_{R \mid C}\right)=$ $k-1-j$ and $h^{0}\left(L_{R \mid D}\right)=j+1$, the inequalities $g(C)+\min \left\{h^{0}\left(L_{R \mid C}\right)-1, i\right\} \leq \operatorname{deg}\left(L_{R \mid C}\right)$ and $g(D)+\min \left\{h^{0}\left(L_{R \mid D}\right)-1, i\right\} \leq \operatorname{deg}\left(L_{R \mid D}\right)$ are satisfied precisely because $j \leq 2 i+2$ and we can invoke Lemma 2.2

Next, for $0 \leq a \leq i$ and $b \geq 1$ we define vector bundles $\mathcal{H}_{a, b}$ over $\widetilde{\mathfrak{G}}_{3 k-6}^{k-2}$ having fibre $\mathcal{H}_{i, j}(C, L)=H^{0}\left(\wedge^{i} M_{\mathbf{P}^{k-2}}(j)\right)$ over each point $(C, L) \in \mathfrak{G}_{3 k-6}^{k-2}$ corresponding to an embedding $C \stackrel{|L|}{\hookrightarrow} \mathbf{P}^{k-2}$.

Recall that we have defined the vector bundles $\mathcal{G}_{0, b}$ over $\widetilde{\mathfrak{G}}_{3 k-6}^{k-2}$. We define the vector bundles $\mathcal{H}_{a, b}$ inductively as follows: $\mathcal{H}_{1, b}$ is defined as the kernel of the multiplication map $\mathcal{G}_{0,1} \otimes \mathcal{G}_{0, b} \rightarrow \mathcal{G}_{0, b+1}$, that is, $\mathcal{H}_{1, b}=\mathcal{G}_{1, b}$. Having already defined $\mathcal{H}_{a-1, b}$ for all $b$, we define $\mathcal{H}_{a, b}$ via the exact sequence

$$
0 \longrightarrow \mathcal{H}_{a, b} \longrightarrow \wedge^{a} \mathcal{G}_{0,1} \otimes \operatorname{Sym}^{b} \mathcal{G}_{0,1} \longrightarrow \mathcal{H}_{a-1, b+1} \longrightarrow 0 .
$$

There is a natural vector bundle morphism $\phi_{a, b}: \mathcal{H}_{a, b} \rightarrow \mathcal{G}_{a, b}$ over $\widetilde{\mathfrak{G}}_{3 k-6}^{k-2}$; when $k=3 i+6, a=i$ and $b=2$, then $\operatorname{rank}\left(\mathcal{H}_{i, b}\right)=\operatorname{rank}\left(\mathcal{G}_{i, b}\right)$ and the degeneracy locus of $\phi_{i, 2}$ is the codimension 1 compactification over $\widetilde{\mathfrak{G}}_{3 k-6}^{k-2}$ of the locus $\mathcal{U}_{g, i}$ defined in Proposition 2.6. 
Next we compute the Chern classes of $\mathcal{H}_{a, b}$ along the curves $X$ and $Y$ :

Proposition 3.14. When $k=3 i+6$, we have the following formulas for the first Chern class of $\mathcal{H}_{i, 2}$ :

$$
c_{1}\left(\mathcal{H}_{i, 2 \mid X}\right)=c_{1}\left(\mathcal{H}_{i, 2 \mid Y}\right)=-(3 i+6)\left(\begin{array}{c}
3 i+4 \\
i
\end{array}\right)((3 i+4) x+\theta) .
$$

Proof. Using repeatedly the sequence (6) we obtain the formula

$$
c_{1}\left(\mathcal{H}_{i, 2 \mid X}\right)=c_{1}\left(\mathcal{H}_{i, 2 \mid Y}\right)=\sum_{l=0}^{i}(-1)^{l} c_{1}\left(\wedge^{i-l} \mathcal{G}_{0,1 \mid X} \otimes \operatorname{Sym}^{l+2} \mathcal{G}_{0,1 \mid X}\right) .
$$

Since $c_{1}\left(\mathcal{G}_{0,1}\right)=-\theta-(3 i+4) x$ and $c_{1}\left(\operatorname{Sym}^{j}\left(\mathcal{G}_{0,1}\right)\right)=\frac{j(3 i+4+j)}{(3 i+5)(3 i+4)}(\underset{j}{3 i+j+3}) c_{1}\left(\mathcal{G}_{0,1}\right)$, the stated formula is obtained after a straightforward calculation.

In the remainder of this section we will compute $c_{1}\left(\mathcal{G}_{i, 2}-\mathcal{H}_{i, 2}\right) \cdot \sigma^{*}\left(C^{j}\right)$, where $C^{j} \subset \Delta_{j}$ is the test curve associated to $[C] \in \mathcal{M}_{2 k-2-j}$. We retain the notation introduced in Propositions 3.4 and 3.5 There are two definitions of the bundles $\mathcal{G}_{a, b \mid \sigma^{*}\left(\Delta_{j}^{0}\right)}$ depending whether $j \leq 2 i+2$ or $j \geq 2 i+3$ and we will explain in detail the calculations only in the first case, the second being similar. We start by describing the Chern number of the restriction of $\mathcal{G}_{0,1}$ to the 1-cycle $\sum_{\alpha=0}^{[j / 2]} a(j, \alpha) X_{j, \alpha}$ on $C \times \operatorname{Pic}^{3 k-j-4}(C)$ :

Proposition 3.15. Suppose $C$ is a general curve of genus $2 k-2-j$ where $2 \leq j \leq 2 i+2$ and let $C^{j} \subset \Delta_{j} \subset \overline{\mathcal{M}}_{2 k-2}$ be the test curve associated to $C$. Then

$$
\begin{gathered}
c_{1}\left(\mathcal{G}_{0,1 \mid \sum_{\alpha=0}^{[j / 2]} a(j, \alpha) X_{j, \alpha}}\right)=-\theta-j((3 k-j-4) \eta+\gamma)-j(j+1)(2 k-3-j) \eta+\aleph, \\
\text { where } \aleph=6(j+1) \frac{\left(\begin{array}{c}
6 i+10-j \\
3 i+6
\end{array}\right)\left(\begin{array}{c}
6 i+8 \\
j
\end{array}\right)}{\left(\begin{array}{c}
3 i+4 \\
j
\end{array}\right)} .
\end{gathered}
$$

Proof. For each $0 \leq \alpha \leq j / 2$, we fix once and for all a linear series $L_{D} \in W_{k-2+j}^{k-2}(D)$ such that $h^{0}\left(L_{D}(-(k+1-\alpha) y)\right)=\alpha$ and $h^{0}\left(L_{D}(-(k+\alpha-j) y)\right)=j-\alpha$ (there are $a(j, \alpha)$ such $\mathfrak{g}_{k-2+j}^{k-2}$ 's and for each of them $X_{j, \alpha} \times\left\{L_{D}\right\}$ is a component of $\left.\sigma^{*}\left(C^{j}\right)\right)$. Just like in Proposition 3.8, we introduce the Poincare bundle $\mathcal{L}$ on $X_{j, \alpha}$ and we define the sheaf on $X_{j, \alpha}$ given by $\mathcal{P}:=\pi_{2}^{*}\left(\pi_{2 *} \mathcal{L}\right)$. Furthermore, for each $a \geq 0$ we consider the Taylor map $\nu_{a}: \mathcal{P} \rightarrow J_{a}(\mathcal{L})$ (the target being the $a$-th jet bundle of $\mathcal{L}$ ), and we set $\mathcal{F}_{a}:=\operatorname{Im}\left(\nu_{a}\right) \subset J_{a}(\mathcal{L})$ and $\mathcal{P}_{a+1}:=\operatorname{Ker}\left(\nu_{a}\right)$.

For each pair $\left(y, L_{C}\right) \in X_{j, \alpha}$ we have an exact sequence

$$
0 \longrightarrow H^{0}\left(D, L_{R \mid D}(-y)\right) \longrightarrow H^{0}\left(C \cup_{y} D, L_{R}\right) \longrightarrow H^{0}\left(C, L_{R \mid C}\right) \longrightarrow 0,
$$

where $L_{R}=\left(L_{C}(-(j+2) y), L_{D}(-(k-2-j) y)\right)$ is the line bundle on $C \cup D$ whose global sections give the fibre of $\mathcal{G}_{0,1}$ at each point in $\widetilde{\mathfrak{G}}_{3 k-6}^{k-2}$. This exact sequence globalizes to an exact sequence of vector bundles on $X_{j, \alpha}$

$$
0 \longrightarrow H^{0}\left(L_{D}(-(k-1-j) y)\right) \otimes \mathcal{O}_{X_{j, \alpha}} \longrightarrow \mathcal{G}_{0,1 \mid X_{j, \alpha}} \longrightarrow \mathcal{P}_{j+2} \longrightarrow 0,
$$

thus $c_{1}\left(\mathcal{G}_{0,1 \mid X_{j, \alpha}}\right)=c_{1}\left(\mathcal{P}_{j+2}\right)$. We now study how the bundles $\mathcal{F}_{a}$ relate to one another via the exact sequences $0 \longrightarrow \pi_{1}^{*}\left(K_{C}^{\otimes a}\right) \otimes \mathcal{L} \longrightarrow J_{a}(\mathcal{L}) \longrightarrow J_{a-1}(\mathcal{L}) \longrightarrow 0$, linking 
successive jet bundles. Recalling that for a generic point $\left(y, L_{C}\right) \in X_{j, \alpha}$ we have that $a^{L_{C}}(y)=(0, \ldots, \alpha-1, \alpha+1, \ldots, j-\alpha, j-\alpha+2, \ldots, k)$ (cf. Proposition 3.4), it follows that $\mathcal{F}_{a}=J_{a}(\mathcal{L})$ for $a \leq \alpha-2$, while $\mathcal{F}_{\alpha-1}$ is obtained from $J_{\alpha-1}(\mathcal{L})$ via an elementary transformation along the divisor $D_{1} \subset X_{j, \alpha}$ consisting of the $b(2 k-2-j, k-2+\alpha-j)$ points $\left(y, L_{C}\right) \in X_{j, \alpha}$ where $a_{\alpha-1}^{L_{C}}(y)=\alpha$. More precisely, one has the exact sequence

$$
0 \longrightarrow \pi_{1}^{*}\left(K_{C}^{\otimes(\alpha-1)}\right) \otimes \mathcal{L} \otimes \mathcal{O}_{X_{j, \alpha}}\left(-D_{1}\right) \longrightarrow \mathcal{F}_{\alpha-1} \longrightarrow J_{\alpha-2}(\mathcal{L}) \longrightarrow 0 .
$$

Furthermore, we have exact sequences

$$
\begin{gathered}
0 \longrightarrow \pi_{1}^{*}\left(K_{C}^{\otimes(\alpha+1)}\right) \otimes \mathcal{L} \longrightarrow \mathcal{F}_{\alpha+1} \longrightarrow \mathcal{F}_{\alpha-1} \longrightarrow 0 \text { and } \\
0 \longrightarrow \pi_{1}^{*}\left(K_{C}^{\otimes a}\right) \longrightarrow \mathcal{F}_{a} \longrightarrow \mathcal{F}_{a-1} \longrightarrow 0, \text { for all } \alpha+2 \leq a \leq j-\alpha-2 .
\end{gathered}
$$

If $D_{2} \subset X_{j, \alpha}$ is the divisor consisting of the $b(2 k-2-j, k-1+\alpha-j)$ points $\left(y, L_{C}\right)$ satisfying the condition $a_{j-\alpha-1}^{L_{C}}(y)=j-\alpha+1$, then we also have the exact sequences

and finally

$$
\begin{gathered}
0 \longrightarrow \pi_{1}^{*}\left(K_{C}^{\otimes(j-\alpha)}\right) \otimes \mathcal{L} \otimes \mathcal{O}_{X_{j, \alpha}}\left(-D_{2}\right) \longrightarrow \mathcal{F}_{j-\alpha} \longrightarrow \mathcal{F}_{j-\alpha-1} \longrightarrow 0 \\
0 \longrightarrow \pi_{1}^{*}\left(K_{C}^{\otimes(j-\alpha+2)}\right) \otimes \mathcal{L} \longrightarrow \mathcal{F}_{j-\alpha+2} \longrightarrow \mathcal{F}_{j-\alpha} \longrightarrow 0
\end{gathered}
$$

$$
0 \longrightarrow \pi_{1}^{*}\left(K_{C}^{\otimes a}\right) \otimes \mathcal{L} \longrightarrow \mathcal{F}_{a} \longrightarrow \mathcal{F}_{a-1} \longrightarrow 0, \text { for } j-\alpha+2 \leq a \leq k-1 .
$$

We gather all the information contained in these sequences to obtain that

$$
\begin{gathered}
c_{1}\left(\mathcal{F}_{j+1}\right)=j c_{1}(\mathcal{L})+j(j+1)(g(C)-1) \eta-\operatorname{deg}\left(D_{1}+D_{2}\right), \text { and then } \\
c_{1}\left(\mathcal{G}_{0,1 \mid \sum_{\alpha} a(j, \alpha) X_{j, \alpha}}\right)=c_{1}(\mathcal{P})-c_{1}\left(\mathcal{F}_{j+1}\right)=-\theta-j((3 k-j-4) \eta+\gamma)-j(j+1)(2 k-3-j) \eta+\aleph, \\
\text { where } \aleph:=\sum_{\alpha=0}^{[j / 2]} a(j, \alpha)(b(2 k-2-j, k-2+\alpha-j)+b(2 k-2-j, k-1+\alpha-j))= \\
=j !(6 i+10-j) ! \sum_{\alpha=0}^{j+1} \frac{(j-2 \alpha-1)(j-2 \alpha)(j-2 \alpha+1)^{2}}{(3 i+5-\alpha) !(3 i+5+\alpha-j) !(j-\alpha+1) ! \alpha !} .
\end{gathered}
$$

This sum can be computed with Maple which finishes the proof.

Next we compute the Chern class of $\mathcal{G}_{0, b}$ with $b \geq 2$. The proof being similar to that in Proposition 3.15 (in fact simpler), we decided to omit it.

Proposition 3.16. Let $b \geq 2$ and $2 \leq j \leq 2 i+2$. Then for each $0 \leq \alpha \leq j / 2$ we have that

$$
c_{1}\left(\mathcal{G}_{0, b \mid X_{j, \alpha}}\right)=-b^{2} \theta-b^{2}(j+2)(\gamma+(3 k-4-j) \eta)-\left(\begin{array}{c}
b j+2 b \\
2
\end{array}\right)(2 k-3-j) \eta .
$$

We turn our attention to the remaining components of $\sigma^{*}\left(C^{j}\right)$. First we show that $Y_{j, \alpha}^{\prime \prime \prime}$ does not appear in the computation of $c_{1}\left(\mathcal{G}_{i, 2}-\mathcal{H}_{i, 2}\right) \cdot \sigma^{*}\left(C^{j}\right)$ :

Proposition 3.17. For $0 \leq \alpha \leq j / 2, a \geq 0$ and $b \geq 1$, we have that $c_{1}\left(\mathcal{G}_{a, b}\right) \cdot Y_{j, \alpha}^{\prime \prime \prime}=$ $c_{1}\left(\mathcal{H}_{a, b}\right) \cdot Y_{j, \alpha}^{\prime \prime \prime}=0$. 
Proof. Clearly, it suffices to show that $c_{1}\left(\mathcal{G}_{0, b}\right) \cdot Y_{j, \alpha}^{\prime \prime \prime}=0$ for all $b \geq 1$ and then use the exact sequences (4) and (6). We carry this out only for $b=1$ the case $b \geq 2$ being analogous. Fix $l=\left(L_{D}, V_{D} \subset H^{0}\left(L_{D}\right)\right) \in Y_{j, \alpha}^{\prime \prime \prime}$. By duality $K_{D} \otimes L_{D}^{\vee}((k+2-\alpha) y)$ is one of the $a(j, \alpha)$ (that is, finitely many) linear systems $\mathfrak{g}_{j-\alpha+1}^{1}$ on $D$ with a $(j-2 \alpha+1)$-fold point at $y$. The space of sections $V_{D} \subset H^{0}\left(L_{D}\right)$ is chosen such that $H^{0}\left(L_{D}(-2 y)\right) \subset V_{D}$, hence $Y_{j, \alpha}^{\prime \prime \prime}$ can be identified with the disjoint union of $a(j, \alpha)$ copies of the projective line $\mathbf{P}\left(H^{0}\left(L_{D}\right) / H^{0}\left(L_{D}(-2 y)\right)\right)$, one for each choice of $L_{D}$. Then $H^{0}\left(L_{C \cup D \mid D}\right)=$ $H^{0}\left(L_{D}(-(k-1-j) y)\right.$ ), for each $l \in Y_{j, \alpha}^{\prime \prime \prime}$ (that is, $\mathcal{G}_{0,1}(l)$ is independent of $\left.V_{D}\right)$. Globalizing this we get that $\mathcal{G}_{0,1 \mid Y_{j, \alpha}^{\prime \prime \prime}}$ is trivial.

In a similar fashion we have the following:

Proposition 3.18. For $0 \leq \alpha \leq j / 2, a \geq 0$ and $b \geq 1$, we have the equalities $c_{1}\left(\mathcal{G}_{a, b}\right) \cdot Y_{j, \alpha}^{\prime}=$ $c_{1}\left(\mathcal{H}_{a, b}\right) \cdot Y_{j, \alpha}^{\prime}=0$.

Proposition 3.19. For $0 \leq \alpha \leq j / 2$ and $b \geq 1$, we have the equality

$$
c_{1}\left(\mathcal{G}_{0, b}\right) \cdot Y_{j, \alpha}^{\prime \prime}=-\frac{b^{2}(j-2 \alpha) j !}{\alpha !(j-\alpha) !} .
$$

Proof. We start by recalling that $Y_{j, \alpha}^{\prime \prime}$ is the locus of line bundles $L_{D} \in \operatorname{Pic}^{k-2+j}(D)$ such that $h^{0}\left(L_{D}(-(k+1+\alpha-j) y)\right) \geq j-\alpha-1$ and $h^{0}\left(L_{D}(-(k-\alpha+1) y)\right) \geq \alpha$. Using [Fu|, Theorem 14.3, one finds that $Y_{j, \alpha}^{\prime \prime} \equiv \frac{j-2 \alpha}{\alpha !(j-\alpha) !} \theta^{j-1}$. Having fixed once and for all one of the finitely many linear series $L_{C} \in W_{3 k-j-4}^{k-2}(C)$ such that $\left\{L_{C}\right\} \times Y_{j, \alpha}^{\prime} \subset \widetilde{\mathfrak{G}}_{3 k-6}^{k-2}$, there is an identification

$$
\mathcal{G}_{0,1}\left(L_{D}\right)=H^{0}\left(L_{D}(-(k-2-j)) y\right),
$$

for each $L_{D} \in Y_{j, \alpha}^{\prime \prime}$. Since the vector bundle $\operatorname{Pic}^{k-2+j}(D) \ni L_{D} \mapsto H^{0}\left(L_{D} \otimes \mathcal{O}_{\beta} y\right)$, is algebraically trivial for each $\beta \geq 0$ (remember that $y \in D$ is a fixed point), we obtain that $c_{1}\left(\mathcal{G}_{0,1 \mid Y_{j, \alpha}^{\prime \prime}}\right)=-\theta$. Similarly, for $b \geq 2$ we get that $c_{1}\left(\mathcal{G}_{0, b \mid Y_{j, \alpha}^{\prime \prime}}\right)=-b^{2} \theta$ from which now the conclusion follows.

Propositions 3.15, 3.16, 3.17, 3.18, 3.19 will enable us to compute the intersection numbers $c_{1}\left(\mathcal{G}_{i, 2}-\mathcal{H}_{i, 2}\right) \cdot \sigma^{*}\left(C^{j}\right)$ needed to determine the coefficient of $\delta_{j}$ in the expansion of $\left[\overline{\mathcal{Z}}_{6 i+10, i}\right]$. Repeatedly using (6) , one can write that

$$
c_{1}\left(\mathcal{H}_{i, 2}\right)=\sum_{l=0}^{i}(-1)^{l} c_{1}\left(\wedge^{i-l} \mathcal{G}_{0,1} \otimes \operatorname{Sym}^{l+2} \mathcal{G}_{0,1}\right)=(3 i+6)\left(\begin{array}{c}
3 i+4 \\
i
\end{array}\right) c_{1}\left(\mathcal{G}_{0,1}\right)
$$

(note the similarity with Proposition 3.14). Using (4) we also have that

$$
c_{1}\left(\mathcal{G}_{i, 2}\right)=\sum_{l=0}^{i}(-1)^{l} c_{1}\left(\wedge^{i-l} \mathcal{G}_{0,1} \otimes \mathcal{G}_{0, l+2}\right),
$$

and a simple calculation yields that

(9) $c_{1}\left(\mathcal{G}_{i, 2}-\mathcal{H}_{i, 2}\right)=-\frac{13 i^{2}+35 i+24}{(3 i+4)(i+1)}\left(\begin{array}{c}3 i+4 \\ i\end{array}\right) c_{1}\left(\mathcal{G}_{0,1}\right)+\sum_{l=0}^{i}(-1)^{l}\left(\begin{array}{c}3 i+5 \\ i-l\end{array}\right) c_{1}\left(\mathcal{G}_{0, l+2}\right)$. 


\section{THE SLOPE OF $\overline{\mathcal{Z}}_{6 i+10, i}$}

In this section we finish the calculation of the class of the virtual divisor $\overline{\mathcal{Z}}_{g, i}$. As before, we fix $i \geq 0, k=3 i+6$ and $g=6 i+10$, hence $\phi: \mathcal{H}_{i, 2} \rightarrow \mathcal{G}_{i, 2}$ is a morphism between two vector bundles of the same rank defined over $\tilde{\mathfrak{G}}_{9 i+12}^{3 i+4}$.

Theorem 4.1. We fix $i \geq 0$ and denote by $\sigma: \widetilde{\mathfrak{G}}_{9 i+12}^{3 i+4} \rightarrow \widetilde{\mathcal{M}}_{6 i+10}$ the natural ptojection map. Then we have the following formula for the class of the pushforward to $\widetilde{\mathcal{M}}_{6 i+10}$ of the virtual degeneracy locus of the morphism $\phi: \mathcal{H}_{i, 2} \rightarrow \mathcal{G}_{i, 2}$ :

$\sigma_{*}\left(c_{1}\left(\mathcal{G}_{i, 2}-\mathcal{H}_{i, 2}\right)\right)=\frac{1}{3 i+5}\left(\begin{array}{c}6 i+7 \\ 3 i+4,2 i+1, i+2\end{array}\right)\left(a \lambda-b_{0} \delta_{0}-\cdots-b_{3 i+4} \delta_{3 i+4}-2 b_{3 i+5} \delta_{3 i+5}\right)$,

where

$$
\begin{gathered}
a=\frac{(4 i+7)\left(6 i^{2}+19 i+12\right)}{(2 i+3)(i+2)}, b_{0}=\frac{12 i^{2}+31 i+18}{3(2 i+3)}, b_{1}=\frac{12 i^{2}+33 i+20}{i+2} \text { and } \\
b_{j}=\frac{j f(i, j)}{6(i+1)(i+2)(2 i+3)(6 i+9-j)}, \text { for } 2 \leq j \leq 2 i+2,
\end{gathered}
$$

with

$$
\begin{gathered}
f(i, j)=864 i^{5}-(240 j-5256) i^{4}+\left(16 j^{2}-998 j+11830\right) i^{3}+ \\
+\left(31 j^{2}-1254 j+11585\right) i^{2}+\left(-5 j^{2}-286 j+3981\right) i-24 j^{2}+240 j-216,
\end{gathered}
$$

and

$$
b_{j}=\frac{2\left(13 i^{2}+35 i+24\right)(3 i+5)}{(i+1)(i+2)\left(\begin{array}{c}
6 i+10 \\
j
\end{array}\right)}\left(\begin{array}{c}
3 i+4 \\
(j-1) / 2
\end{array}\right)^{2}+\frac{g(i, j)}{12(i+1)(i+2)(2 i+3)(6 i+9-j)}
$$

for an odd $j$ such that $2 i+3 \leq j \leq 3 i+5$, where

$$
\begin{gathered}
g(i, j)=1728 j i^{5}-\left(576 j^{2}-10512 j+540\right) i^{4}+\left(48 j^{3}-2592 j^{2}+24120 j-3234\right) i^{3}+ \\
\left(140 j^{3}-3926 j^{2}+25176 j-7278\right) i^{2}+\left(109 j^{3}-2107 j^{2}+10875 j-7263\right) i+12 j^{3}-156 j^{2}+972 j-2700
\end{gathered}
$$

and

$$
b_{j}=\frac{\left(13 i^{2}+35 i+24\right)\left((3 i+5)(2 j+1)-j^{2}\right)}{2(i+1)(i+2)(3 i+5)\left(\begin{array}{c}
6 i+10 \\
j
\end{array}\right)}\left(\begin{array}{c}
3 i+5 \\
j / 2
\end{array}\right)^{2}+\frac{h(i, j)}{12(i+1)(i+2)(2 i+3)(6 i+9-j)},
$$

for an even $j$ such that $2 i+3 \leq j \leq 3 i+5$, where

$$
\begin{gathered}
g(i, j)=1728 j i^{5}-\left(576 j^{2}-10512 j+1020\right) i^{4}+\left(48 j^{3}-2592 j^{2}+24280 j-6034\right) i^{3}+ \\
\left(140 j^{3}-3942 j^{2}+25830 j-13400\right) i^{2}+\left(109 j^{3}-2145 j^{2}+11774 j-13230\right) i+12 j^{3}-180 j^{2}+1392 j-4896 .
\end{gathered}
$$

In particular, $b_{j} \geq b_{0}$ for all $1 \leq j \leq 3 i+5$ and

$$
s\left(\sigma_{*}\left(c_{1}\left(\mathcal{G}_{i, 2}-\mathcal{H}_{i, 2}\right)\right)\right)=\frac{a}{b_{0}}=\frac{3(4 i+7)\left(6 i^{2}+19 i+12\right)}{\left(12 i^{2}+31 i+18\right)(i+2)} .
$$


Proof. Since codim $\left(\overline{\mathcal{M}}_{g}-\widetilde{\mathcal{M}}_{g}, \overline{\mathcal{M}}_{g}\right) \geq 2$, it makes no difference whether we compute the class $\sigma_{*}\left(\mathcal{G}_{i, 2}-\mathcal{H}_{i, 2}\right)$ on $\widetilde{\mathcal{M}}_{g}$ or on $\overline{\mathcal{M}}_{g}$ and we can write

$$
\sigma_{*}\left(\mathcal{G}_{i, 2}-\mathcal{H}_{i, 2}\right)=A \lambda-B_{0} \delta_{0}-B_{1} \delta_{1}-\cdots-B_{3 i+5} \delta_{3 i+5} \in \operatorname{Pic}\left(\overline{\mathcal{M}}_{g}\right),
$$

where $\lambda, \delta_{0}, \ldots, \delta_{3 i+5}$ are the generators of $\operatorname{Pic}\left(\overline{\mathcal{M}}_{g}\right)$. We start with the following:

Claim: One has the relation $A-12 B_{0}+B_{1}=0$.

We pick a general curve $[C, q] \in \mathcal{M}_{6 i+9,1}$ and at the fixed point $q$ we attach to $C$ a Lefschetz pencil of plane cubics. If we denote by $R \subset \overline{\mathcal{M}}_{g}$ the resulting curve, then $R \cdot \lambda=1, R \cdot \delta_{0}=12, R \cdot \delta_{1}=-1$ and $R \cdot \delta_{j}=0$ for $j \geq 2$. The relation $A-12 B_{0}+B_{1}=0$ follows once we show that $\sigma^{*}(R) \cdot c_{1}\left(\mathcal{G}_{i, 2}-\mathcal{H}_{i, 2}\right)=0$. To achieve this we check that $\mathcal{G}_{0, b \mid \sigma^{*}(R)}$ is trivial and then use (4) and (6). We take $\left[C \cup_{q} E\right]$ to be an arbitrary curve from $R$, where $E$ is an elliptic curve. Using that limit $\mathfrak{g}_{9 i+12}^{3 i+4}$ on $C \cup_{q} E$ are in $1: 1$ correspondence with linear series $L \in W_{9 i+12}^{3 i+4}(C)$ having a cusp at $q$ (this being a statement that holds independent of $E$ ) and that $\mathcal{G}_{0, b \mid \sigma^{*}\left(\Delta_{1}^{0}\right)}$ consists on each fibre of sections of the genus $g-1$ aspect of the limit $\mathfrak{g}_{9 i+12}^{3 i+4}$, the claim now follows.

Now we determine $A, B_{0}$ and $B_{1}$ explicitly. We fix a general pointed curve $(C, q) \in$ $\mathcal{M}_{6 i+9,1}$ and construct the test curves $C^{1} \subset \Delta_{1}$ and $C^{0} \subset \Delta_{0}$. Using the notation from Proposition 3.3. we get that $\sigma^{*}\left(C^{0}\right) \cdot c_{1}\left(\mathcal{G}_{i, 2}-\mathcal{H}_{i, 2}\right)=c_{1}\left(\mathcal{G}_{i, 2 \mid Y}\right)-c_{1}\left(\mathcal{H}_{i, 2 \mid Y}\right)$ and $\sigma^{*}\left(C^{1}\right) \cdot c_{1}\left(\mathcal{G}_{i, 2}-\mathcal{H}_{i, 2}\right)=c_{1}\left(\mathcal{G}_{i, 2 \mid X}\right)-c_{1}\left(\mathcal{H}_{i, 2 \mid X}\right)$ (the other component $T$ of $\sigma^{*}\left(C^{1}\right)$ does not appear because $\mathcal{G}_{0, b \mid T}$ is trivial for all $b \geq 1$ ). On the other hand

$C^{0} \cdot \sigma_{*}\left(c_{1}\left(\mathcal{G}_{i, 2}-\mathcal{H}_{i, 2}\right)\right)=(12 i+18) B_{0}-B_{1}$ and $C^{1} \cdot \sigma_{*}\left(c_{1}\left(\mathcal{G}_{i, 2}-\mathcal{H}_{i, 2}\right)\right)=(12 i+16) B_{1}$,

while we already know that $A-12 B_{0}+B_{1}=0$. Using Propositions 3.11, 3.12, 3.14, the expressions for $[X]$ and $[Y]$ as well as the well-known formula $\theta^{j} x^{k-2-j}=g ! /(g-j)$ ! on $C_{k-2}$, we get a linear system of 3 equations in $A, B_{0}$ and $B_{1}$ which leads to the stated formulas for the first three coefficients.

To compute $B_{j}$ for $2 \leq j \leq 3 i+5$ we fix general curves $[C] \in \mathcal{M}_{6 i+10-j}$ and $[D, y] \in \mathcal{M}_{j, 1}$ and consider the test curve $C^{j} \subset \Delta_{j}$. Then

$\sigma^{*}\left(C^{j}\right) \cdot c_{1}\left(\mathcal{G}_{i, 2}-\mathcal{H}_{i, 2}\right)=c_{1}\left(\mathcal{G}_{i, 2}-\mathcal{H}_{i, 2}\right) \cdot \sum_{\alpha=0}^{[j / 2]}\left(\left(a(j, \alpha) X_{j, \alpha}+b(6 i+10-j, 3 i+5+\alpha-j) Y_{j, \alpha}^{\prime \prime}\right)\right.$.

Using (9) together with Proposition 3.19 , we get that

$$
\begin{gathered}
2(6 i+9-j) B_{j}=c_{1}\left(\mathcal{G}_{i, 2}-\mathcal{H}_{i, 2}\right) \cdot \sum_{\alpha=0}^{[j / 2]} b(6 i+10-j, 3 i+5+\alpha-j) Y_{j, \alpha}^{\prime \prime}= \\
=\frac{\left(8 i^{2}+19 i+12\right) j !(6 i+10-j) !(3 i+2) !}{i !(2 i+4) !}\left(\sum_{\alpha=0}^{j} \frac{(j-2 \alpha-1)(j-2 \alpha)^{2}(j-2 \alpha+1)}{\alpha !(j-\alpha) !(3 i+5-\alpha) !(3 i+5+\alpha-j) !}\right),
\end{gathered}
$$

which can be computed by Maple, whereas $c_{1}\left(\mathcal{G}_{i, 2}-\mathcal{H}_{i, 2}\right) \cdot\left(\sum_{\alpha=0}^{[j / 2]} a(j, \alpha) X_{j, \alpha}\right)$ can be computed using (9) and Propositions 3.15 and 3.16. The rest now follows and checking that $b_{j} \geq b_{0}$ for $j \geq 1$ is elementary. 
Theorem 4.2. Assume that vector bundle morphism $\phi: \mathcal{H}_{i, 2} \rightarrow \mathcal{G}_{i, 2}$ is non-degenerate at a general point from $\sigma^{-1}\left(\Delta_{1}^{0}\right)$. Then $\overline{\mathcal{Z}}_{6 i+10, i}$ is a divisor on $\overline{\mathcal{M}}_{6 i+10}$ and

$$
6<s\left(\overline{\mathcal{Z}}_{6 i+10, i}\right)=\frac{3(4 i+7)\left(6 i^{2}+19 i+12\right)}{\left(12 i^{2}+31 i+18\right)(i+2)}<6+\frac{12}{g+1} .
$$

Remark 4.3. Since $\sigma^{-1}\left(\Delta_{1}^{0}\right)$ is irreducible, to verify the assumption made in Theorem 4.2 it suffices to show that if $C \subset \mathbf{P}^{3 i+4}$ is a general 1-cuspidal curve with $p_{a}(C)=6 i+10$ and $\operatorname{deg}(C)=9 i+12$, then $C$ satisfies property $\left(N_{i}\right)$. We checked this for $g=16,22$ (cf. Remarks 2.10 and 2.12), while the case $g=10$ was treated in [FP]. The assumption in Theorem 4.2 implies that $\phi$ is non-degenerate over a general point from $\sigma^{-1}\left(\Delta_{0}^{0}\right)$ and also over a general point from $\sigma^{-1}\left(\mathcal{M}_{g}^{0}\right)$, which is equivalent to $\mathcal{Z}_{g, i}$ being a divisor on $\mathcal{M}_{g}^{0}$. Therefore the assumption is slightly stronger than Conjecture 2.7

Proof of Theorem 4.2 We denote by $Z(\phi)$ the degeneracy locus of $\phi$, thus $\sigma_{*}(Z(\phi))$ is a divisor on $\overline{\mathcal{M}}_{6 i+10, i}$ such that $\sigma_{*}(Z(\phi))=\overline{\mathcal{Z}}_{g, i}+\sum_{j=0}^{3 i+5} d_{j} \Delta_{j}$, for certain coefficients $d_{j} \geq 0$. Using the Remark above we obtain that $d_{0}=d_{1}=0$, hence $s\left(\overline{\mathcal{Z}}_{6 i+10, i}\right)=$ $s\left(\sigma_{*}\left(c_{1}\left(\mathcal{G}_{i, 2}-\mathcal{H}_{i, 2}\right)\right)\right)$ and the rest follows from Theorem 4.1

Remark 4.4. In the simplest case $i=0$, we can compare the formula for $\left[\overline{\mathcal{Z}}_{6 i+10, i}\right]$ with our findings in $[\mathrm{FP}]$. Theorem 4.1 gives the formula

$$
\sigma_{*}\left(c_{1}\left(\mathcal{G}_{0,2}-\mathcal{H}_{0,2}\right)\right) \equiv 42\left(7 \lambda-\delta_{0}-5 \delta_{1}-\frac{4}{3} \delta_{2}-7 \delta_{3}-\frac{44}{7} \delta_{4}-\frac{685}{42} \delta_{5}\right),
$$

whereas Theorem 1.6 from $[\mathrm{FP}]$ says that if $\mathcal{K}_{10}$ is the divisor on $\mathcal{M}_{10}$ of curves lying on a $K 3$ surface then

$$
\overline{\mathcal{K}}_{10}=7 \lambda-\delta_{0}-5 \delta_{1}-9 \delta_{2}-12 \delta_{3}-14 \delta_{4}-b_{5} \delta_{5},
$$

where $b_{5} \geq 6$. Since we have also established the set-theoretic equality $\mathcal{Z}_{10,0}=\mathcal{K}_{10}$ (cf. $[\mathrm{FP}]$, Theorem 1.7), it follows that there is a scheme-theoretic equality $\mathcal{Z}_{10,0}=42 \mathcal{K}_{10}$. Here 42 is the number of pencils $\mathfrak{g}_{6}^{1}$ on a general curve of genus 10 , and its appearance in the formula of $\left[\overline{\mathcal{Z}}_{10,0}\right]$ has a clear geometric meaning: if a Brill-Noether general curve $[C] \in \mathcal{M}_{10}$ fails $\left(N_{0}\right)$ for one linear system $\mathfrak{g}_{12}^{4}=K_{C}\left(-\mathfrak{g}_{6}^{1}\right)$, then it fails $\left(N_{0}\right)$ for all 42 linear systems $\mathfrak{g}_{12}^{4}$ it possesses, that is, the map $\sigma: \mathcal{U}_{10,0} \rightarrow \mathcal{K}_{10,0}$ is $42: 1$. Moreover, the vector bundle morphism $\mathcal{H}_{0,2} \rightarrow \mathcal{G}_{0,2}$ is degenerate along each of the boundary divisors $\Delta_{2}, \ldots, \Delta_{5}$. This situation presumably extends to higher genera as well hence the main thrust of Theorem 4.1 is that it computes $s\left(\overline{\mathcal{Z}}_{6 i+10, i}\right)$.

To finish the proof of Theorems 1.1 and 1.2 we specialize to the cases $g=16$ and 22 and note that the assumption made in Theorem 4.2 is satisfied in these situations (cf. Theorems 2.8 and 2.11.

\section{AN EFFECTIVE DIVISOR ON $\overline{\mathcal{M}}_{14,1}$}

In this section we describe how to construct effective divisors on $\overline{\mathcal{M}}_{g, n}$ using syzygy type conditions for pointed curves. We treat only one example. We denote 
by $\overline{\mathcal{Z}}_{14,0}^{1}$ the closure in $\overline{\mathcal{M}}_{14,1}$ of the locus $\mathcal{Z}_{14,0}^{1}$ of smooth pointed curves $[C, p] \in \mathcal{M}_{14,1}$ for which there exists a linear series $L \in W_{18}^{6}(C)$ such that the map

$$
\mu_{L, p}: \operatorname{Sym}^{2} H^{0}(C, L(-p)) \rightarrow H^{0}\left(C, L^{\otimes 2}(-2 p)\right)
$$

is not an isomorphism. Just like the in the case of the loci $\mathcal{Z}_{g, i}$ on $\mathcal{M}_{g}$, the locus $\mathcal{Z}_{14,0}^{1}$ can be naturally viewed as the pushforward of the degeneracy locus of a morphism between two vector bundles of the same rank 21 over $\mathcal{G}_{18}^{6} \times{ }_{\mathcal{M}_{14}} \mathcal{M}_{14,1}$.

We are going to show that $\overline{\mathcal{Z}}_{14,0}^{1}$ is a divisor and in order to compute its class we need some preparations. Recall that for $g \geq 3$, the group $\operatorname{Pic}\left(\overline{\mathcal{M}}_{g, 1}\right)$ is freely generated by $\lambda$, the tautological class $\psi$ and the boundary classes $\delta_{i}=\left[\Delta_{i}\right]$ with $0 \leq i \leq g-1$, where for $i \geq 1$, the generic point of $\Delta_{i}$ is a union of two smooth curves of genus $i$ and $g-i$ meeting at a point, the marked point lying on the genus $i$ component. We denote by $\pi: \overline{\mathcal{M}}_{g, 1} \rightarrow \overline{\mathcal{M}}_{g}$ the natural forgetful map. We can then write the class of $\overline{\mathcal{Z}}_{14,0}^{1}$ on $\overline{\mathcal{M}}_{14,1}$ as

$$
\overline{\mathcal{Z}}_{14,0}^{1} \equiv a \lambda+c \psi-b_{0} \delta_{0}-b_{1} \delta_{1}-\cdots-b_{13} \delta_{13}
$$

As before, we will determine the relevant coefficients in the expression of $\left[\overline{\mathcal{Z}}_{14,0}^{1}\right]$ by intersecting our divisor with various test curves.

Proposition 5.1. Let $C$ be a general curve of genus 14 and $\widetilde{C}=\pi^{-1}([C]) \subset \overline{\mathcal{M}}_{14,1}$, the test curve obtained by letting the marked point vary along $C$. Then $\widetilde{C} \cdot \overline{\mathcal{Z}}_{14,0}^{1}=12012$, hence $c=\widetilde{C} \cdot \overline{\mathcal{Z}}_{14,0}^{1} /(2 g-2)=462$.

Proof. Let us fix a linear series $L \in W_{18}^{6}(C)$. We count the number of points $p \in C$, for which the multiplication map $\mu_{L, p}$ not injective. If $p_{1}: C \times C \rightarrow C$ and $p_{2}: C \times C \rightarrow C$ are the two projections, we define the vector bundles

$$
\mathcal{E}:=\left(p_{2}\right)_{*}\left(p_{1}^{*}(L) \otimes \mathcal{O}_{C \times C}(-\Delta)\right) \text { and } \mathcal{F}:=\left(p_{2}\right)_{*}\left(p_{1}^{*}\left(L^{\otimes 2}\right) \otimes \mathcal{O}_{C \times C}(-2 \Delta)\right) .
$$

There is a natural multiplication map $\mu_{L}: \operatorname{Sym}^{2}(\mathcal{E}) \rightarrow \mathcal{F}$, and the cardinality of its degeneracy locus is just $c_{1}(\mathcal{F})-c_{1}\left(\operatorname{Sym}^{2}(\mathcal{E})\right)$. A simple calculation shows that $c_{1}(\mathcal{E})=$ $-4 x-\theta=-18$, hence $c_{1}\left(\operatorname{Sym}^{2}(\mathcal{E})\right)=-126$, while $c_{1}(\mathcal{F})=-98$. We obtain that $\mu_{L, p}$ is not an isomorphism for precisely 28 points $p \in C$. Since $C$ has 429 linear series $\mathfrak{g}_{18}^{6}$ (cf. [ACGH], we obtain $c=\left(\tilde{C} \cdot \overline{\mathcal{Z}}_{14,0}^{1}\right) / 26=429 \cdot 28 / 26=462$.

For more relations among the coefficients of $\left[\overline{\mathcal{Z}}_{14,0}^{1}\right]$ we define the map $\nu: \overline{\mathcal{M}}_{1,2} \rightarrow \overline{\mathcal{M}}_{g, 1}$ obtained by attaching to each 2-pointed elliptic curve $[E, q, p]$ a fixed general 1-pointed curve $[C, q] \in \mathcal{M}_{g-1}$ (the point of attachment being $q$ ). One has the pullback formulas

$$
\nu^{*}(\lambda)=\lambda, \nu^{*}(\psi)=\psi_{p}, \nu^{*}\left(\delta_{0}\right)=\delta_{0}, \nu^{*}\left(\delta_{1}\right)=-\psi_{q} \text { and } \nu^{*}\left(\delta_{g-1}\right)=\delta_{q p},
$$

where $\psi_{p}$ and $\psi_{q}$ are the tautological classes corresponding to the marked points $p$ and $q$, while $\delta_{q p}$ is the boundary component of curves having a rational tail containing both $q$ and $p$. On $\overline{\mathcal{M}}_{1,2}$ these classes are not independent and we have the relations $\psi_{q}=\psi_{p}$, $\lambda=\psi_{p}-\delta_{q p}$ and $\delta_{0}=12\left(\psi_{p}-\delta_{q p}\right)$ (see e.g. [AC2], Proposition 1.9). 
Proposition 5.2. If $\nu: \overline{\mathcal{M}}_{1,2} \rightarrow \overline{\mathcal{M}}_{14,1}$ is as above, then $\nu^{*}\left(\overline{\mathcal{Z}}_{14,0}^{1}\right)=\emptyset$. It follows that we have the relations

$$
a-12 b_{0}+b_{13}=0 \text { and } c+b_{1}=b_{13} .
$$

Proof. We assume that $\left[X=C \cup_{q} E, p \in E\right] \in \overline{\mathcal{Z}}_{14,0}^{1}$. Then there exists a limit linear series $\mathfrak{g}_{18}^{6}$ on $X$ determined by its aspects $L_{C} \in W_{18}^{6}(C)$ and $L_{E} \in W_{18}^{6}(E)$, together with compatible elements

$\rho_{C} \in \operatorname{Ker}\left\{\mu_{L_{C}}: \operatorname{Sym}^{2} H^{0}\left(L_{C}\right) \rightarrow H^{0}\left(L_{C}^{\otimes 2}\right)\right\}, \rho_{E} \in \operatorname{Ker}\left\{\mu_{L_{E}}: \operatorname{Sym}^{2} H^{0}\left(L_{E}\right) \rightarrow H^{0}\left(L_{E}^{\otimes 2}\right)\right\}$, satisfying the inequality

$$
\operatorname{ord}_{q}\left(\rho_{C}\right)+\operatorname{ord}_{q}\left(\rho_{E}\right) \geq \operatorname{deg}\left(L_{C}\right)+\operatorname{deg}\left(L_{E}\right)=36
$$

and such that $\rho_{E} \in \operatorname{Sym}^{2} H^{0}\left(L_{E}(-p)\right.$ ) (see $|\overline{F P}|$, Section 4 , for how to study multiplication maps in the context of limit linear series). Because $(C, q) \in \overline{\mathcal{M}}_{13,1}$ is general, the vanishing sequence of $L_{C}$ at $q$ equals $(0,2,3,4,5,6,7)$, the vanishing sequence of $L_{E}$ at $q$ is $(11,12,13,14,15,16,18)$ and finally, the vanishing sequence of $L_{E}$ at $p$ is either $(0,1,2,3,4,5,6)$ or $(0,1,2,3,4,5,7)$, depending on whether $p-q \in \operatorname{Pic}^{0}(E)$ is a 7 -torsion class or not.

We claim that $\operatorname{ord}_{q}\left(\rho_{E}\right) \leq 29(=13+16=14+15)$. Indeed, otherwise $\operatorname{ord}_{q}\left(\rho_{E}\right) \geq$ $30(=14+16=15+15)$, and since $\rho_{E} \in \operatorname{Sym}^{2} H^{0}\left(L_{E}(-p)\right)$, after subtracting base points $\rho_{E}$ becomes $\mathrm{a} \neq 0$ element in the kernel of the map $\operatorname{Sym}^{2} H^{0}(N) \rightarrow H^{0}\left(N^{\otimes 2}\right)$, where $N=L_{E}(-p-14 q) \in \operatorname{Pic}^{3}(E)$. This is obviously impossible. Therefore ord $\operatorname{ord}_{q}\left(\rho_{E}\right) \leq 29$, so by compatibility, $\operatorname{ord}_{q}\left(\rho_{C}\right) \geq 7(=2+5=3+4)$. We now show that when $(C, p) \in \mathcal{M}_{13,1}$ is chosen generically, there can be no such element $\rho_{C}$ which leads to a contradiction.

Claim: Suppose $\sigma_{0}, \sigma_{2}, \sigma_{3}, \sigma_{4}, \sigma_{5}, \sigma_{6}, \sigma_{7}$ is a basis of $H^{0}\left(L_{C}\right)$ adapted to the point $q$ in the sense that $\operatorname{ord}_{q}\left(\sigma_{i}\right)=i$. If $W\left(q, L_{C}\right) \subset \operatorname{Sym}^{2} H^{0}\left(L_{C}\right)$ denotes the 17-dimensional subspace spanned by the elements $\sigma_{i} \cdot \sigma_{j}$ with $4 \leq i \leq j \leq 7, \sigma_{3} \cdot \sigma_{j}$ for $j \geq 4$ and $\sigma_{2} \cdot \sigma_{j}$ for $j \geq 5$, then the restriction of the multiplication map $W\left(q, L_{C}\right) \rightarrow H^{0}\left(L_{C}^{2}(-7 q)\right)$ is an isomorphism. (Note that $W\left(q, L_{C}\right)$ does not depend on the chosen basis $\left\{\sigma_{i}\right\}$ ).

The proof of this claim is similar to the proof of Theorem 5.1 in $|\mathrm{FP}|$. It is enough to construct a single 1-cuspidal curve $X \subset \mathbf{P}^{6}$ with $p_{a}(X)=14$ and $\operatorname{deg}(X)=18$, such that if $\nu: C \rightarrow X$ is the normalization of $X$ and $q \in C$ is the inverse image of the cusp, then $X$ does not lie on any quadric contained in $W\left(q, \nu^{*}\left(\mathcal{O}_{X}(1)\right)\right)$. We construct the following cuspidal curve: define $\Gamma \subset \mathbf{P}^{6}$ to be the image of the map $t \stackrel{f}{\mapsto}$ $\left[1, t^{2}, t^{3}, t^{4}, t^{5}, t^{6}, t^{7}\right]$, then choose a general hyperplane $H \subset \mathbf{P}^{6}$ which intersects $\Gamma$ in distinct points $p_{1}, \ldots, p_{7}$. Take $D \subset H$ to be a general smooth curve of genus $g(D)=7$ and $\operatorname{deg}(D)=11$ which passes through $p_{1}, \ldots, p_{7}$. Then $X:=\Gamma \cup D$ is a curve of arithmetic genus 14 and degree 21 having a cusp at the point $q=f(0) \in \Gamma$. The quadrics in $W\left(q, \nu^{*}\left(\mathcal{O}_{X}(1)\right)\right)$ can of course be written down explicitly and to show that $D$ can be chosen such that it is not contained in any quadric from $W\left(q, \nu^{*}\left(\mathcal{O}_{X}(1)\right)\right)$ amounts to a simple counting argument.

Remark 5.3. The claim we have just proved also shows that $\overline{\mathcal{Z}}_{14,0}^{1}$ is a divisor on $\overline{\mathcal{M}}_{14,1}$. Alternatively, this can be proved in the same way as Theorem 2.8 
We now determine the coefficient $b_{1}$ :

Proposition 5.4. Consider general curves $[E, p, q] \in \mathcal{M}_{1,2}$ and $[C] \in \mathcal{M}_{13}$, and denote by $C^{2} \subset \overline{\mathcal{M}}_{14,1}$ the test curve consisting of points $\left\{\left[C \cup_{q} E, p \in E\right]\right\}_{\{q \in C\}}$. Then $C^{2} \cdot \overline{\mathcal{Z}}_{14,0}^{1}=$ 133848 , therefore $b_{1}=C^{2} \cdot \overline{\mathcal{Z}}_{14,0}^{1} / 24=5577$.

Proof. Throughout the proof we will use the notations introduced in Proposition 3.11, From the proof of Proposition 5.2 it is clear that the intersection number $C^{2} \cdot \overline{\mathcal{Z}}_{14,0}^{1}$ equals the number of pairs $(q, D) \in X \subset C \times C_{6}$ such that the map

$$
W\left(q, K_{C}(-D)\right) \rightarrow H^{0}\left(K_{C}^{\otimes 2}(-2 D-7 q)\right)
$$

is not an isomorphism. To compute this number we construct the rank 17 vector bundle $W$ over $X$ having fibre $W(q, D)=W\left(q, K_{C}(-D)\right)$ over each point $(q, D) \in X$. The curve $C$ being general, the vanishing sequence $a^{K_{C}(-D)}(q)$ will be generically equal to $(0,2,3,4,5,6,7)$, while at a finite number of points $(q, D) \in X$ we will have that $a^{K_{C}(-D)}(q)=(0,2,3,4,5,6,8)$. In order to compute $c_{1}(W)$ we note that $W$ has a subbundle $W_{1} \subset W$ which fits into two exact sequences:

$$
0 \longrightarrow \operatorname{Sym}^{2} u_{*}\left(v^{*}(\mathcal{M}) \otimes \mathcal{O}(-4 \Delta)\right)_{\mid X} \longrightarrow W_{1} \rightarrow u_{*}\left(v^{*}(\mathcal{M}) \otimes \mathcal{O}(-4 \Delta)\right)_{\mid X} \otimes \mathcal{P} \longrightarrow 0,
$$

where $\mathcal{P}=u_{*}\left(v^{*}(\mathcal{M}) \otimes \mathcal{I}_{\Delta}^{3} / \mathcal{I}_{\Delta}^{4}\right)_{\mid X^{\prime}}$ and

$$
0 \longrightarrow W_{1} \longrightarrow W \longrightarrow u_{*}\left(v^{*}(\mathcal{M}) \otimes \mathcal{O}(-5 \Delta)\right)_{\mid X} \otimes u_{*}\left(v^{*}(\mathcal{M}) \otimes \mathcal{I}_{\Delta}^{2} / \mathcal{I}_{\Delta}^{3}\right)_{\mid X} \longrightarrow 0 .
$$

Next, we consider the multiplication map $W \rightarrow u_{*}\left(v^{*}\left(\mathcal{M}^{\otimes 2}\right) \otimes \mathcal{O}(-7 \Delta)\right)_{\mid X}$ whose degeneration locus we want to compute. The intersection number $C^{2} \cdot \overline{\mathcal{Z}}_{14,0}^{1}$ is equal to

$$
\left(c_{1}\left(u_{*}\left(v^{*}\left(\mathcal{M}^{\otimes 2}\right) \otimes \mathcal{O}(-7 \Delta)\right)\right)-c_{1}(W)\right) \cdot[X]=(3 \theta-7 x-\gamma+18 \eta) \cdot[X]=133848,
$$

as it turns out after a short calculation. Here we have used that $c_{1}(W)$ can be computed from the two exact sequences involving $W$ and $W_{1}$, while

$$
c_{1}\left(u_{*}\left(v^{*}\left(\mathcal{M}^{\otimes 2}\right) \otimes \mathcal{O}(-7 \Delta)\right)\right)=c_{1}\left(\mathcal{F}_{2}\right)-c_{1}\left(J_{6}\left(\mathcal{M}^{\otimes 2}\right)\right)=-4 \theta-34 x+14 \gamma-756 \eta .
$$

Since $b_{1}=5577$ we now obtain that $b_{13}=b_{1}+c=6039$. To compute the coefficient $b_{0}$ (and thus the $\lambda$-coefficient $a$ ) we use our last test curve:

Proposition 5.5. Let $(C, q, p) \in \mathcal{M}_{13,2}$ be a general 2-pointed curve. We denote by $C^{3} \subset$ $\overline{\mathcal{M}}_{14,1}$ the family consisting of curves $\{[C / y \sim q, p]\}_{\{y \in C\}}$. Then $C^{3} \cdot \overline{\mathcal{Z}}_{14,0}^{1}=c+26 b_{0}-b_{13}=$ 24453. It follows that $b_{0}=1155$ and $a=7821$.

Proof. We retain the notations introduced in Proposition 3.12 We construct a vector bundle map $\operatorname{Sym}^{2}(\mathcal{E}) \rightarrow \mathcal{F}$ over the curve $Y \subset C \times C_{6}$, where $\mathcal{E}=u_{*}\left(v^{*}(\mathcal{M}) \otimes \mathcal{O}\left(-\Gamma_{p}\right)\right)$ is the bundle with fibre $\mathcal{E}(y, D)=H^{0}\left(K_{C}(-D-p)\right)$, while $\mathcal{F}$ is the bundle with fibre

$$
\mathcal{F}(y, D)=H^{0}\left(K_{C}^{\otimes 2}(-2 D-2 p-y-q)\right) \oplus \mathbb{C} \cdot t^{2} \subset H^{0}\left(K_{C}^{\otimes^{2}}(-2 D-2 p)\right),
$$

where $H^{0}\left(K_{C}(-D-p)\right) / H^{0}\left(K_{C}(-D-p-y-q)\right)=\mathbb{C} \cdot t$, for every $(y, D) \in Y$. 
It is easy to show that $c_{1}(\mathcal{E})=-5 x-\theta$, hence $c_{1}\left(\operatorname{Sym}^{2}(\mathcal{E})\right)=-35 x-7 \theta$. The class $c_{1}(\mathcal{F})$ can be computed from the exact sequence

$0 \longrightarrow u_{*}\left(v^{*}\left(\mathcal{M}^{\otimes 2}\right) \otimes \mathcal{O}\left(-2 \Gamma_{p}-\Gamma_{q}-\Delta\right)\right)_{\mid Y} \longrightarrow \mathcal{F} \longrightarrow\left(\mathcal{M}^{\otimes 2} \otimes \mathcal{O}\left(-2 \Gamma_{p}\right)\right)_{\mid Y}(-A) \longrightarrow 0$,

where $A=Y \cap \pi_{1}^{-1}(p)$ is the effective divisor on the curve $Y$ consisting of all points $(p, D)$ such that $h^{0}(p+q+D) \geq 2$. Using the formula for $[Y]$ (cf. Proposition 3.12), we get that $|A|=429$. We also compute that $c_{1}\left(u_{*}\left(v^{*}\left(\mathcal{M}^{\otimes 2}\right) \otimes \mathcal{O}\left(-2 \Gamma_{p}-\Gamma_{q}-\Delta\right)\right)\right)=$ $-4 \theta-40 x-33 \eta+2 \gamma$ and of course $c_{1}\left(\mathcal{M}^{\otimes 2} \otimes \mathcal{O}\left(-2 \Gamma_{p}\right)\right)=2(17 \eta-\gamma-x)$. Therefore we can write that

$$
C^{3} \cdot \overline{\mathcal{Z}}_{14,0}^{1}=\left(c_{1}(\mathcal{F})-c_{1}\left(\operatorname{Sym}^{2}(\mathcal{E})\right)\right) \cdot[Y]-|A|=(-7 x+3 \theta+\eta) \cdot[Y]-|A|=24453 .
$$

We have thus far determined the coefficients $a, c, b_{0}, b_{1}, b_{13}$ in the expansion of $\left[\overline{\mathcal{Z}}_{14,0}^{1}\right]$. This is already enough to conclude that $\left[\overline{\mathcal{Z}}_{14,0}^{1}\right]$ lies outside the cone of $\operatorname{Pic}\left(\overline{\mathcal{M}}_{14,1}\right)$ spanned by pullbacks of effective divisors from $\overline{\mathcal{M}}_{14}$, Brill-Noether divisors on $\overline{\mathcal{M}}_{14,1}$ and boundary divisors (see the discussion after Theorem 1.4 for the relevance of this result). To get a bound on the remaining coefficients $b_{j}$ for $2 \leq j \leq 12$, we use a variant of Theorem 1.1 from [FP]. The boundary divisor $\Delta_{j} \subset \overline{\mathcal{M}}_{14,1}$ with $3 \leq j \leq 12, j \neq 4$ is filled-up by pencils $R_{j}$ obtained by attaching to a fixed 2-pointed curve $[B, p, q] \in \mathcal{M}_{j, 2}$ a variable 1-pointed curve $[C, q] \in \overline{\mathcal{M}}_{14-j, 1}$ moving in a Lefschetz pencils of curves of genus $14-j$ sitting on a fixed $K 3$ surface. Deformations of $R_{j}$ cover $\Delta_{j}$ for $j \neq 2,4$, hence we have that $R_{j} \cdot \overline{\mathcal{Z}}_{14,0}^{1} \geq 0$. Since one also has the relations (see $|\mathrm{FP}|$, Lemma 2.4)

$$
R_{j} \cdot \lambda=15-j, R_{j} \cdot \delta_{j}=-1, R_{j} \cdot \delta_{0}=6(17-j), R_{j} \cdot \psi=0 \text { and } R_{j} \cdot \delta_{i}=0 \text { for } i \neq 0, j,
$$

we immediately get the estimate $b_{j} \geq 15+27 j$ for all $3 \leq j \leq 12, j \neq 4$. To obtain the bounds $b_{2} \geq 325$ and $b_{4} \geq 271$ we use similar pencils filling up $\Delta_{2}$ and $\Delta_{4}$ respectively. We skip these details. This completes the proof of Theorem 1.4

\section{THE KODAIRA DIMENSION OF $\overline{\mathcal{M}}_{g, n}$}

In this last section we use the effective divisors $\overline{\mathcal{Z}}_{16,1}$ and $\overline{\mathcal{Z}}_{22,2}$ to improve Logan's results about which moduli spaces $\overline{\mathcal{M}}_{g, n}$ are of general type. For a general reference

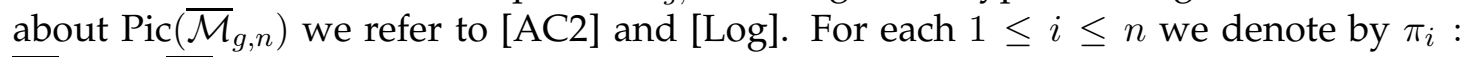
$\overline{\mathcal{M}}_{g, n} \rightarrow \overline{\mathcal{M}}_{g, 1}$ the morphism forgetting all marked points except the one labelled by $i$ and we also consider the map $\pi: \overline{\mathcal{M}}_{g, n} \rightarrow \overline{\mathcal{M}}_{g}$ which forgets all marked points. We recall that the canonical class of $\overline{\mathcal{M}}_{g, n}$ is given by the formula

$$
K_{\overline{\mathcal{M}}_{g, n}}=13 \lambda-2 \delta_{0}+\sum_{i=1}^{n} \psi_{i}-2 \sum_{i \geq 0, S} \delta_{i: S}-\sum_{S} \delta_{1: S} .
$$

Here $\psi_{i}$ is the tautological class corresponding to the $i$-th marked point, while $\delta_{i: S}$ with $i \geq 0$ and $\emptyset \neq S \subset\{1, \ldots, n\}$ denotes the class of the boundary divisor with generic point being a union of two curves of genus $i$ and $g-i$ such that the marked points on the genus $i$ component are precisely those labelled by $S$. 
Proof of Theorem 1.3 We start with the case $g=22$ and we show that $\overline{\mathcal{M}}_{22,2}$ is of general type. On $\overline{\mathcal{M}}_{22,2}$ we consider the averaged pullback of the Weierstrass divisor

$$
W_{12}:=\pi_{1}^{*}(\overline{\mathcal{W}})+\pi_{2}^{*}(\overline{\mathcal{W}}) \equiv-2 \lambda+\frac{g(g+1)}{2}\left(\psi_{1}+\psi_{2}\right)-\sum_{i \geq 0, S \neq \emptyset} c_{i: S} \delta_{i: S},
$$

where $c_{i: S} \geq 0$, and significantly, the coefficient of $\delta_{0}$ is 0 . By Theorem 1.2 we have another effective class, namely $\left[\pi^{*}\left(\overline{\mathcal{Z}}_{22,2}\right)\right]=c\left(\frac{1665}{256} \lambda-\delta_{0}-\cdots\right)$, where $c>0$. One can easily check that $K_{\overline{\mathcal{M}}_{22,2}}$ can be written as a positive combination of $\left[W_{12}\right],\left[\pi^{*}\left(\overline{\mathcal{Z}}_{22,2}\right)\right], \psi_{1}+\psi_{2}$ and some other boundary classes. Since $\psi_{1}+\psi_{2}$ is big and nef, it follows that $\overline{\mathcal{M}}_{22,2}$ is of general type.

When $g=21$ we use the maps $\chi_{i, j}: \overline{\mathcal{M}}_{21,5} \rightarrow \overline{\mathcal{M}}_{22}$ for $1 \leq i<j \leq 5$, where $\chi_{i, j}$ identifies the marked points labelled by $i$ and $j$ and forgets those labelled by $\{i, j\}^{c}$. The $\mathbb{Q}$-divisor class

$$
\sum_{i<j} \chi_{i, j}^{*}\left(\overline{\mathcal{Z}}_{22,2}\right) \equiv c\left(\frac{1665}{256} \lambda-\delta_{0}+\frac{2}{5} \sum_{i=1}^{5} \psi_{i}-\cdots\right), \text { where } c>0,
$$

is obviously effective on $\overline{\mathcal{M}}_{21,5}$. Since the $\mathbb{Q}$-class $-\lambda+11 \sum_{i=1}^{5} \psi_{i}-0 \cdot \delta_{0}-\cdots$, is also effective (cf. |Log|, Theorem 5.4 - we have retained only the coefficients that play a role in our argument), once again we see that $K_{\overline{\mathcal{M}}_{21,5}}$ can be written as the sum of an effective divisor and a positive multiple of $\sum_{i=1}^{5} \psi_{i}$.

Finally we settle the case $g=16$ : we adapt Theorem 5.4 from |Log| to conclude that the $\mathbb{Q}$-class $-\lambda+\frac{23}{9}\left(\sum_{i=1}^{9} \psi_{i}\right)-0 \cdot \delta_{0}-\cdots$, is effective on $\overline{\mathcal{M}}_{16,9}$ (precisely, this is the class of the $S_{9}$-orbit of the closure in $\overline{\mathcal{M}}_{16,9}$ of the effective divisor $D$ on $\mathcal{M}_{16,9}$ consisting of points $\left[C, p_{1}, \ldots, p_{9}\right]$ such that $h^{0}\left(C, 2 p_{1}+\cdots+2 p_{7}+p_{8}+p_{9}\right) \geq 2$ and we have explicitly indicated that the coefficient of $\delta_{0}$ is 0 and retained only the coefficients that are significant for this calculation). The class $\left[\pi^{*}\left(\overline{\mathcal{Z}}_{16,1}\right)\right]=c\left(\frac{407}{61} \lambda-\delta_{0}-\cdots\right)$, where $c>0$, is also effective and one writes $K_{\overline{\mathcal{M}}_{16,9}}$ as a positive sum of these two effective classes, boundary classes and the big and nef class $\sum_{i=1}^{9} \psi_{i}$.

Remark 6.1. Theorem 6.3 from [Log] claims that $\overline{\mathcal{M}}_{22,4}$ is of general type but the numerical argument used in the proof seems to be incorrect.

\section{REFERENCES}

[AC1] E. Arbarello and M. Cornalba, Footnotes to a paper of Beniamino Segre, Math. Ann. 256 (1981), 341-362.

[AC2] E. Arbarello and M. Cornalba, Calculating cohomology groups of moduli spaces of curves via algebraic geometry, Inst. Hautes Etudes Sci. Publ. Math. 88 (1998), 97-127.

[ACGH] E. Arbarello, M. Cornalba, P. Griffiths and J. Harris, Geometry of algebraic curves, Grundlehren der mathematischen Wissenschaften 267, Springer.

[EH1] D. Eisenbud and J. Harris, Limit linear series: basic theory, Invent. Math. 85 (1986), 337-371.

[EH2] D. Eisenbud and J. Harris, Irreducibility of some families of linear series with Brill-Noether number -1 , Ann. Scient. Ec. Norm. Sup.(4) 22 (1989), 33-53.

[EH3] D. Eisenbud and J. Harris, The Kodaira dimension of the moduli space of curves of genus $\geq 23$, Invent. Math. 90 (1987), 359-387.

[F] G. Farkas, The geometry of the moduli space of curves of genus 23, Math. Ann. 318 (2000), 43-65. 
[FP] G. Farkas and M. Popa, Effective divisors on $\overline{\mathcal{M}}_{g}$, curves on K3 surfaces and the Slope Conjecture, J. Algebraic Geometry, 14 (2005), 241-267.

[Fu] W. Fulton, Intersection theory, Ergebnisse der Mathematik und ihrer Grenzgebiete 2, Springer 1998.

[GL] M. Green and R. Lazarsfeld, Some results on syzygies of finite sets and algebraic curves, Compositio Math. 67 (1988), 301-314.

[HM] J. Harris and D. Mumford, On the Kodaira dimension of the moduli space of curves, Invent. Math. 67 (1982), 23-88.

[HMo] J. Harris and I. Morrison, Slopes of effective divisors on the moduli space of stable curves, Invent. Math. 99 (1990), 321-355.

[L] R. Lazarsfeld, A sampling of vector bundle techniques in the study of linear series, in: Lectures on Riemann Surfaces, (M. Cornalba, X. Gomez-Mont, A. Verjovsky eds.), World Scientific 1989, 500-559.

[Kh] D. Khosla, Harvard Ph.D. Thesis (2005).

[Log] A. Logan, The Kodaira dimension of moduli spaces of curves with marked points, Amer. J. of Math. 125 (2003), 105-138.

[SchT] F.-O. Schreyer and F. Tonoli, Needles in a Haystack: Special Varieties via Small Fields, in: Computations in Algebraic Geometry with Macaulay 2, Algorithms and Computations in Math. 8 (2002) 251-279.

[V1] C. Voisin, Sur l'application de Wahl des courbes satisfaisant la condition de Brill-Noether-Petri, Acta Math. 168 (1992), 249-272.

[V2] C. Voisin, Green's generic syzygy conjecture for curves of even genus lying on a K3 surface, J. Eur. Math. Soc. 4 (2002), 363-404.

[V3] C. Voisin, Green's canonical syzygy conjecture for generic curves of odd genus, math.AG/0301359 to appear in Compositio Math.

Department of MATHEMAtics, University of TeXas, Austin, TX 78712

E-mail address: gfarkas@math.utexas.edu 